Journal of Engineering and Applied Sciences 15 (2): 679-693, 2020

ISSN: 1816-949X

(C) Medwell Journals, 2020

\title{
Asymptotic Behavior of Eigenvalue and Eigenfunction of a Six Order Boundary Value Problem
}

\author{
Karwan H.F. Jwamer and Khelan H. Qadr \\ Department of Mathematics, College of Science, University of Sulaimani, \\ Kurdistan Region, Sulaymaniyah, Iraq
}

\begin{abstract}
We consider differential operators with separate boundary conditions in this study. And we find a new expression and their derivative of six linearly independent solution. And it will also prove the existence of uniqueness for separate boundary conditions. We obtain asymptotic formulas for the individual values and functions of these problems with boundary value where the potential $q(x)$ is an arbitrary complex function valued in $[\mathrm{a}, \mathrm{b}]$.
\end{abstract}

Key words: Differential operator, eigenvalue, eigenfunctions, spectral parameter, arbitrary complex function, potential

\section{INTRODUCTION}

It is well known that many researchers have investigated the spectral properties of the Sturm-Liouville operator generated by the separate boundary condition (Aigunov, 1996) and many researchers have found asymptotic formula for the Sturm-Liouville operator's eigenvalues and functions in the case of periodic andanti-periodic boundary conditions (Menken, 2010; Naimark, 1967; Moller and Zinsou, 2012; Jwamer and Aigounv, 2010; Aigounov and Tamila, 2009; Aigunov, 1996 and Tamarkin, 1928). Many researchers have been interested in the ongoing Sturm-Liouville issue in recent years as we see N.B. Kermov, H. Menken by Menken (2010), Karwan and Rando (2017) found upper bound with smooth coefficients for the proper functions of the fourth boundary value issue. In this study, we consider the differential operator:

$$
\begin{gathered}
y^{(6)}(x)+q(x) y(x)=\lambda^{6} y(x) \\
U_{i}(y)=\sum_{j=0}^{5} a_{i j} y^{(j)}(b, \lambda), i=0,1,2
\end{gathered}
$$

where, $\mathrm{a}_{\mathrm{ij}}$ are real numbers:

$$
\begin{array}{r}
\mathrm{U}_{3}(\mathrm{y})=\sum_{\mathrm{l}=1}^{6}(\lambda)^{\mathrm{l}-1} \mathrm{y}^{(6-\imath)}(\mathrm{b}, \lambda) \\
\mathrm{U}_{4}(\mathrm{y})=\sum_{\mathrm{l}=1}^{6}(-\lambda)^{\mathrm{l}-1} \mathrm{y}^{(6-\imath)}(\mathrm{b}, \lambda)
\end{array}
$$

$$
\mathrm{U}_{5}(\mathrm{y})=\sum_{\mathrm{i}=1}^{6}\left(\frac{\lambda}{\left(\frac{1}{2}+\mathrm{i} \frac{\sqrt{3}}{2}\right)}\right)^{\mathrm{l}-1} \mathrm{y}^{(6-\imath)}(\mathrm{b}, \lambda)
$$

Where:

$\lambda \quad$ : A spectral parameter

$\mathrm{q}(\mathrm{x})$ : An arbitrary complex valued function

\section{FUNDAMENTAL DIFFERENTIAL EQUATION SOLUTIONS SYSTEMS}

The expressions of six linearly independent solutions and their derivatives can be found in this study.

Theorem 1: The fundamental system of solutions of linear differential equation:

$$
y^{(6)}(x)+q(x) y(x)=\lambda^{6} y(x)
$$

Are $\mathrm{y}_{0}(\mathrm{x}, \lambda), \mathrm{y}_{1}(\mathrm{x}, \lambda), \mathrm{y}_{2}(\mathrm{x}, \lambda), \mathrm{y}_{3}(\mathrm{x}, \lambda), \mathrm{y}_{4}(\mathrm{x}, \lambda), \mathrm{y}_{5}$ $(\mathrm{x}, \lambda)$ that satisfy the initial conditions:

$$
y_{i}^{(n)}(0, \lambda)= \begin{cases}1 & \text { if } i=n \\ 0 & \text { if } i \neq n\end{cases}
$$

Where:

$$
\begin{aligned}
& \mathrm{y}_{0}=\frac{1}{3}\left[\cosh \lambda \mathrm{x}+\cosh \lambda\left(\frac{1}{2}+\mathrm{i} \frac{\sqrt{3}}{2}\right) \mathrm{x}+\cosh \lambda\left(-\frac{1}{2}+\mathrm{i} \frac{\sqrt{3}}{2}\right) \mathrm{x}\right]+\frac{1}{3 \lambda^{5}} \\
& \int_{0}^{\mathrm{x}}\left[\sinh \lambda(\mathrm{x}-\xi)+\sinh \lambda\left(\frac{1}{2}+\mathrm{i} \frac{\sqrt{3}}{2}\right)(\mathrm{x}-\xi)+\sinh \lambda\left(-\frac{1}{2}+\mathrm{i} \frac{\sqrt{3}}{2}\right)(\mathrm{x}-\xi)\right] \\
& \mathrm{q}(\xi) \mathrm{y}_{0}(\xi) \mathrm{d} \xi
\end{aligned}
$$




$$
\begin{aligned}
& \mathrm{y}_{1}=\left[\frac{1}{3 \lambda} \sinh \lambda \mathrm{x}+\frac{1}{6 \lambda}\left(1+\frac{1}{32} \mathrm{i}\right) \sinh \lambda\left(\frac{1}{2}+\mathrm{i} \frac{\sqrt{3}}{2}\right) \mathrm{x}+\frac{1}{6 \lambda}\left(-1+\frac{1}{32} \mathrm{i}\right) \sinh \lambda\left(-\frac{1}{2}+\mathrm{i} \frac{\sqrt{3}}{2}\right) \mathrm{x}\right]+\frac{1}{3 \lambda^{5}} \\
& \int_{\mathrm{a}}^{\mathrm{b}}\left[\sinh \lambda(\mathrm{x}-\xi)+\sinh \lambda\left(\frac{1}{2}+\mathrm{i} \frac{\sqrt{3}}{2}\right)(\mathrm{x}-\xi)+\sinh \lambda\left(-\frac{1}{2}+\mathrm{i} \frac{\sqrt{3}}{2}\right)(\mathrm{x}-\xi)\right] \mathrm{q}(\xi) \mathrm{y}_{0}(\xi) \mathrm{d} \xi \\
& \mathrm{y}_{2}=\left[\frac{1}{3 \lambda^{2}} \cosh \lambda \mathrm{x}+\frac{1}{6 \lambda^{2}}\left(-1+\frac{1}{32} \mathrm{i}\right) \cosh \lambda\left(\frac{1}{2}+\mathrm{i} \frac{\sqrt{3}}{2}\right) \mathrm{x}+\frac{1}{6 \lambda^{2}}\left(1-\frac{1}{32} \mathrm{i}\right) \cosh \lambda\left(-\frac{1}{2}+\mathrm{i} \frac{\sqrt{3}}{2}\right) \mathrm{x}\right]+\frac{1}{3 \lambda^{5}} \\
& \int_{0}^{x}\left[\sinh \lambda(x-\xi)+\left(\frac{1}{2}+i \frac{\sqrt{3}}{2}\right) \sinh \lambda\left(\frac{1}{2}+i \frac{\sqrt{3}}{2}\right)(x-\xi)+\left(-\frac{1}{2}+i \frac{\sqrt{3}}{2}\right) \sinh \lambda\left(-\frac{1}{2}+i \frac{\sqrt{3}}{2}\right)(x-\xi)\right] q(\xi) y_{0}(\xi) d \xi \\
& \mathrm{y}_{3}=\frac{1}{3 \lambda^{3}}\left[\sinh \lambda \mathrm{x}-\sinh \lambda\left(\frac{1}{2}+\mathrm{i} \frac{\sqrt{3}}{2}\right) \mathrm{x}+\sinh \lambda\left(-\frac{1}{2}+\mathrm{i} \frac{\sqrt{3}}{2}\right) \mathrm{x}\right]+\frac{1}{3 \lambda^{5}} \\
& \int_{0}^{x}\left[\sinh \lambda(x-\xi)+\sinh \lambda\left(\frac{1}{2}+i \frac{\sqrt{3}}{2}\right)(x-\xi)+\sinh \lambda\left(-\frac{1}{2}+i \frac{\sqrt{3}}{2}\right)(x-\xi)\right] q(\xi) y_{0}(\xi) d \xi \\
& \mathrm{y}_{4}=\left[\frac{1}{3 \lambda^{4}} \cosh \lambda \mathrm{x}+\frac{1}{6 \lambda^{4}}\left(1-\frac{1}{32} \mathrm{i}\right) \cosh \lambda\left(\frac{1}{2}+\mathrm{i} \frac{\sqrt{3}}{2}\right) \mathrm{x}+\frac{1}{6 \lambda^{4}}\left(-1+\frac{1}{32} \mathrm{i}\right) \cosh \lambda\left(-\frac{1}{2}+\mathrm{i} \frac{\sqrt{3}}{2}\right) \mathrm{x}\right]+\frac{1}{3 \lambda^{5}} \\
& \int_{0}^{x}\left[\sinh \lambda(x-\xi)+\left(\frac{1}{2}+i \frac{\sqrt{3}}{2}\right) \sinh \lambda\left(\frac{1}{2}+i \frac{\sqrt{3}}{2}\right)(x-\xi)+\left(-\frac{1}{2}+i \frac{\sqrt{3}}{2}\right) \sinh \lambda\left(-\frac{1}{2}+i \frac{\sqrt{3}}{2}\right)(x-\xi)\right] q(\xi) y_{0}(\xi) d \xi \\
& \mathrm{y}_{5}=\left[\frac{1}{3 \lambda^{5}} \sinh \lambda \mathrm{x}-\frac{1}{6 \lambda^{5}}\left(-1+\frac{1}{32} \mathrm{i}\right) \sinh \lambda\left(\frac{1}{2}+\mathrm{i} \frac{\sqrt{3}}{2}\right) \mathrm{x}-\frac{1}{6 \lambda^{5}}\left(1+\frac{1}{32} \mathrm{i}\right) \sinh \lambda\left(-\frac{1}{2}+\mathrm{i} \frac{\sqrt{3}}{2}\right) \mathrm{x}\right]+\frac{1}{3 \lambda^{5}} \\
& \int_{0}^{x}\left[\sinh \lambda(x-\xi)+\left(\frac{1}{2}+i \frac{\sqrt{3}}{2}\right) \sinh \lambda\left(\frac{1}{2}+i \frac{\sqrt{3}}{2}\right)(x-\xi)+\left(-\frac{1}{2}+i \frac{\sqrt{3}}{2}\right) \sinh \lambda\left(-\frac{1}{2}+i \frac{\sqrt{3}}{2}\right)(x-\xi)\right] q(\xi) y_{0}(\xi) d \xi
\end{aligned}
$$

Proof: Consider the linear differential operator:

$$
l(y)=-y^{(6)}(x)+q(x) y(x)
$$

We want to find a solution that is not zero:

$$
1(y)-\lambda^{6} y(x)=0
$$

Which satisfy the initial conditions Eq. 2. First, we reduce Eq. 3 to an integro-differential equations:

$$
\begin{gathered}
y^{(6)}(x)+\lambda^{6} y(x)=q(x) y(x) \\
m(y)=y^{(6)}(x)+\lambda^{6} y(x), m(y)=q(x) y(x)
\end{gathered}
$$

The homogeneous linear differential equation $y^{(6)}(x)+\lambda^{6} y(x)=0$ has for $\lambda \neq 0$ the solutions:

$$
e^{\lambda w_{0} x}, e^{\lambda w_{1} x}, e^{\lambda w_{2} x}, e^{\lambda w_{3} x}, e^{\lambda w_{4} x}, e^{\lambda w_{5} x}
$$

Where:

$$
\begin{aligned}
& \mathrm{w}_{0}=1, \mathrm{w}_{1}=\frac{1}{2}+\mathrm{i} \frac{\sqrt{3}}{2}, \mathrm{w}_{2}=-\frac{1}{2}+\mathrm{i} \frac{\sqrt{3}}{2}, \\
& \mathrm{w}_{3}=-1, \mathrm{w}_{4}=-\frac{1}{2}-\mathrm{i} \frac{\sqrt{3}}{2}, \mathrm{w}_{5}=\frac{1}{2}-\mathrm{i} \frac{\sqrt{3}}{2}
\end{aligned}
$$

Then by using the method of variation of parameters we can express the solutions of Eq. 4 for $\mathrm{k}=0,1,2,3,4$, 5 as:

$$
\begin{aligned}
& \mathrm{y}_{\mathrm{k}}=\mathrm{c}_{0} \mathrm{e}^{\lambda \mathrm{x}}+\mathrm{c}_{1} \mathrm{e}^{\lambda\left(\frac{1}{2}+\mathrm{i} \frac{\sqrt{3}}{2}\right) \mathrm{x}}+\mathrm{c}_{2} \mathrm{e}^{\lambda\left(\frac{1}{2}+\mathrm{i} \frac{\sqrt{3}}{2}\right) \mathrm{x}}+ \\
& c_{3} e^{-\lambda x}+c_{4} e^{\lambda\left(-\frac{1}{2}-i \frac{\sqrt{3}}{2}\right) x}+c_{5} e^{\lambda\left(\frac{1}{2}-i \frac{\sqrt{3}}{2}\right) x}+ \\
& \sinh (x-\xi)+\left(\frac{1}{2}+i \frac{\sqrt{3}}{2}\right) \sinh \lambda\left(\frac{1}{2}+i \frac{\sqrt{3}}{2}\right)(x-\xi)+ \\
& \int_{0}^{x} \frac{\left(-\frac{1}{2}+i \frac{\sqrt{3}}{2}\right) \sinh \lambda\left(-\frac{1}{2}+i \frac{\sqrt{3}}{2}\right)(x-\xi)}{3 \lambda^{5}} \\
& q(\xi) y_{k}(x, \lambda) d \xi
\end{aligned}
$$

We apply Eq. 5 and in Eq. 2, then, we get. For $\mathrm{k}=0$ then: 


$$
\begin{aligned}
& \mathrm{y}_{0}(0, \lambda)=1, \mathrm{y}_{0}^{\prime}(0, \lambda)=0, \mathrm{y}_{0}^{\prime \prime}(0, \lambda)=0, \\
& \mathrm{y}_{0}^{\prime \prime \prime}(0, \lambda)=0, \mathrm{y}_{0}^{(4)}(0, \lambda)=0, \mathrm{y}_{0}^{(5)}(0, \lambda)=0 \\
& \mathrm{c}_{0}+\mathrm{c}_{1}+\mathrm{c}_{2}+\mathrm{c}_{3}+\mathrm{c}_{4}+\mathrm{c}_{5}=1 \\
& \lambda c_{0}+\left(\frac{1}{2}+i \frac{\sqrt{3}}{2}\right) \lambda c_{1}+\left(-\frac{1}{2}+i \frac{\sqrt{3}}{2}\right) \lambda c_{2}- \\
& \lambda \mathrm{c}_{3}+\left(-\frac{1}{2}-\mathrm{i} \frac{\sqrt{3}}{2}\right) \lambda \mathrm{c}_{4}+\left(\frac{1}{2}-\mathrm{i} \frac{\sqrt{3}}{2}\right) \lambda \mathrm{c}_{5}=0 \\
& \lambda^{2} \mathrm{c}_{0}+\left(\frac{1}{2}+\mathrm{i} \frac{\sqrt{3}}{2}\right)^{2} \lambda^{2} \mathrm{c}_{1}+\left(-\frac{1}{2}+\mathrm{i} \frac{\sqrt{3}}{2}\right)^{2} \lambda^{2} \mathrm{c}_{2}+ \\
& \lambda^{2} \mathrm{c}_{3}+\left(-\frac{1}{2}-\mathrm{i} \frac{\sqrt{3}}{2}\right)^{2} \lambda^{2} \mathrm{c}_{4}+\left(\frac{1}{2}-\mathrm{i} \frac{\sqrt{3}}{2}\right)^{2} \lambda^{2} \mathrm{c}_{5}=0 \\
& \lambda^{3} \mathrm{c}_{0}+\left(\frac{1}{2}+\mathrm{i} \frac{\sqrt{3}}{2}\right)^{3} \lambda^{3} \mathrm{c}_{1}+\left(-\frac{1}{2}+\mathrm{i} \frac{\sqrt{3}}{2}\right)^{3} \lambda^{3} \mathrm{c}_{2}-\lambda^{3} \mathrm{c}_{3}+ \\
& \left(-\frac{1}{2}-\mathrm{i} \frac{\sqrt{3}}{2}\right)^{3} \lambda^{3} \mathrm{c}_{4}+\left(\frac{1}{2}-\mathrm{i} \frac{\sqrt{3}}{2}\right)^{3} \lambda^{3} \mathrm{c}_{5}=0 \\
& \lambda^{4} \mathrm{c}_{0}+\left(\frac{1}{2}+\mathrm{i} \frac{\sqrt{3}}{2}\right)^{4} \lambda^{4} \mathrm{c}_{1}+\left(-\frac{1}{2}+\mathrm{i} \frac{\sqrt{3}}{2}\right)^{4} \lambda^{4} \mathrm{c}_{2}+ \\
& \lambda^{4} \mathrm{C}_{3}+\left(-\frac{1}{2}-\mathrm{i} \frac{\sqrt{3}}{2}\right)^{4} \lambda^{4} \mathrm{C}_{4}+\left(\frac{1}{2}-\mathrm{i} \frac{\sqrt{3}}{2}\right)^{4} \lambda^{4}{ }^{4} \mathrm{C}_{5}=0 \\
& \lambda^{5} c_{0}+\left(\frac{1}{2}+\mathrm{i} \frac{\sqrt{3}}{2}\right)^{5} \lambda^{5} c_{1}+\left(-\frac{1}{2}+\mathrm{i} \frac{\sqrt{3}}{2}\right)^{5} \lambda^{5} c_{2}-\lambda^{5} c_{3}+ \\
& \left(-\frac{1}{2}-\mathrm{i} \frac{\sqrt{3}}{2}\right)^{5} \lambda^{5} \mathrm{c}_{4}+\left(\frac{1}{2}-\mathrm{i} \frac{\sqrt{3}}{2}\right)^{5} \lambda^{5} \mathrm{c}_{5}=0
\end{aligned}
$$

We can solve it for $c_{i}$ and we get $c_{i}=1 / 6$ for each $\mathrm{k}=0: 5$ then $\mathrm{y}_{0}$ has the form:

$$
\begin{aligned}
& y_{0}=\frac{1}{3}\left[\cosh \lambda x+\cosh \lambda\left(\frac{1}{2}+i \frac{\sqrt{3}}{2}\right) x+\cosh \lambda\left(-\frac{1}{2}+i \frac{\sqrt{3}}{2}\right) x\right]+\frac{1}{3 \lambda^{5}} \\
& \int_{0}^{x}\left[\begin{array}{l}
\left.\sinh \lambda(x-\xi)+\sinh \lambda\left(\frac{1}{2}+i \frac{\sqrt{3}}{2}\right)\right] q(\xi) y_{0}(\xi) d \xi \\
(x-\xi)+\sinh \lambda\left(-\frac{1}{2}+i \frac{\sqrt{3}}{2}\right)(x-\xi)
\end{array}\right]
\end{aligned}
$$

And we can use the same technique for $\mathrm{y}_{1}(\mathrm{x}, \lambda)$, $\mathrm{y}_{2}(\mathrm{x}, \lambda), \mathrm{y}_{3}(\mathrm{x}, \lambda), \mathrm{y}_{4}(\mathrm{x}, \lambda), \mathrm{y}_{5}(\mathrm{x}, \lambda)$.

Corollary 1: For $x$ in $[a, b]$ and $\lambda \neq 0, y_{0}(x, \lambda)$ and its derivatives in theorem 1 can be written as:

$$
\begin{aligned}
& \mathrm{y}_{0}=\frac{1}{3} \\
& {\left[\begin{array}{l}
\cosh \lambda(x-a)+\cosh \lambda\left(\frac{1}{2}+i \frac{\sqrt{3}}{2}\right)(x-a)+ \\
\cosh \lambda\left(-\frac{1}{2}+i \frac{\sqrt{3}}{2}\right)(x-a)
\end{array}\right]+0\left(\frac{1}{\lambda^{5}} e^{t t \mid(x-a)}\right)} \\
& \mathrm{y}_{0}^{\prime}=\frac{1}{3} \lambda\left[\begin{array}{l}
\sinh \lambda(\mathrm{x}-\mathrm{a})+\left(\frac{1}{2}+\mathrm{i} \frac{\sqrt{3}}{2}\right) \sinh \lambda\left(\frac{1}{2}+\mathrm{i} \frac{\sqrt{3}}{2}\right) \\
(\mathrm{x}-\mathrm{a})+\left(-\frac{1}{2}+\mathrm{i} \frac{\sqrt{3}}{2}\right) \sinh \lambda\left(-\frac{1}{2}+\mathrm{i} \frac{\sqrt{3}}{2}\right)(\mathrm{x}-\mathrm{a})
\end{array}\right]+ \\
& 0\left(\frac{1}{\lambda^{4}} \mathrm{e}^{|\mathrm{t}|(\mathrm{x}-\mathrm{a})}\right) \\
& y_{0}^{\prime \prime}=\frac{1}{3} \lambda^{2} \\
& {\left[\begin{array}{l}
\cosh \lambda(\mathrm{x}-\mathrm{a})+\left(\frac{1}{2}+\mathrm{i} \frac{\sqrt{3}}{2}\right) \cosh \lambda\left(\frac{1}{2}+\mathrm{i} \frac{\sqrt{3}}{2}\right)(\mathrm{x}-\mathrm{a})+ \\
\left(-\frac{1}{2}+\mathrm{i} \frac{\sqrt{3}}{2}\right) \cosh \lambda\left(-\frac{1}{2}+\mathrm{i} \frac{\sqrt{3}}{2}\right)(\mathrm{x}-\mathrm{a})
\end{array}\right]+} \\
& 0\left(\frac{1}{\lambda^{3}} \mathrm{e}^{|\mathrm{t}|(\mathrm{x}-\mathrm{a})}\right) \\
& y_{0}^{\prime \prime \prime}=\frac{1}{3} \lambda^{3} \\
& {\left[\begin{array}{l}
\sinh \lambda(x-a)+\left(\frac{1}{2}+i \frac{\sqrt{3}}{2}\right) \sinh \lambda\left(\frac{1}{2}+\mathrm{i} \frac{\sqrt{3}}{2}\right)(x-a)+ \\
\left(-\frac{1}{2}+i \frac{\sqrt{3}}{2}\right) \sinh \lambda\left(-\frac{1}{2}+i \frac{\sqrt{3}}{2}\right)(x-a)
\end{array}\right]+} \\
& 0\left(\frac{1}{\lambda^{2}} \mathrm{e}^{|\mathrm{t}|(\mathrm{x}-\mathrm{a})}\right)
\end{aligned}
$$$$
\begin{aligned}
& \mathrm{y}_{0}^{(4)}=\frac{1}{3} \lambda^{4}\left[\begin{array}{l}
\cosh \lambda(\mathrm{x}-\mathrm{a})+\left(\frac{1}{2}+\mathrm{i} \frac{\sqrt{3}}{2}\right) \cosh \lambda\left(\frac{1}{2}+\mathrm{i} \frac{\sqrt{3}}{2}\right) \\
(\mathrm{x}-\mathrm{a})+\left(-\frac{1}{2}+\mathrm{i} \frac{\sqrt{3}}{2}\right) \cosh \lambda\left(-\frac{1}{2}+\mathrm{i} \frac{\sqrt{3}}{2}\right)(\mathrm{x}-\mathrm{a})
\end{array}\right]+ \\
& 0\left(\frac{1}{\lambda} \mathrm{e}^{|\mathrm{t}|(\mathrm{x}-\mathrm{a})}\right)
\end{aligned}
$$$$
y_{0}^{(5)}=\frac{1}{3} \lambda^{5}\left[\begin{array}{l}
\sinh \lambda(x-a)+\left(\frac{1}{2}+i \frac{\sqrt{3}}{2}\right) \sinh \lambda\left(\frac{1}{2}+i \frac{\sqrt{3}}{2}\right) \\
(x-a)+\left(-\frac{1}{2}+i \frac{\sqrt{3}}{2}\right) \sinh \lambda\left(-\frac{1}{2}+i \frac{\sqrt{3}}{2}\right)(x-a)
\end{array}\right]+
$$$$
0\left(e^{|t|(x-a)}\right)
$$

Proof: Since, $\mathrm{y}_{0}(\mathrm{x}, \lambda)$ in theorem 1 has the form: 


$$
\begin{aligned}
& y_{0}=\frac{1}{3}\left[\cosh \lambda(x-a)+\cosh \lambda\left(\frac{1}{2}+i \frac{\sqrt{3}}{2}\right)(x-a)+\cosh \lambda\left(-\frac{1}{2}+i \frac{\sqrt{3}}{2}\right)(x-a)\right]+\frac{1}{3 \lambda^{5}} \\
& \int_{a}^{x}\left[\sinh \lambda(x-\xi)+\left(\frac{1}{2}+i \frac{\sqrt{3}}{2}\right) \sinh \lambda\left(\frac{1}{2}+i \frac{\sqrt{3}}{2}\right)(x-\xi)+\left(-\frac{1}{2}+i \frac{\sqrt{3}}{2}\right) \sinh \lambda\left(-\frac{1}{2}+i \frac{\sqrt{3}}{2}\right)(x-\xi)\right] \\
& q(\xi) y_{0}(\xi) d \xi
\end{aligned}
$$

Since, $|\cosh \mathrm{z}| \leq \mathrm{e}^{|\operatorname{re~}(z)|}$ and $|\sinh \mathrm{z}| \leq \mathrm{e}^{|\operatorname{re~}(z)|}$ :

$$
\begin{gathered}
|\sinh \lambda(x-\xi)| \leq \mathrm{e}^{|\sigma(x-\xi)|},\left|\sinh \lambda\left(\frac{1}{2}+\mathrm{i} \frac{\sqrt{3}}{2}\right)(\mathrm{x}-\xi)\right| \leq \mathrm{e}^{\left|\left(\frac{1}{2} \sigma-\frac{\sqrt{3}}{2} \mathrm{t}\right)(\mathrm{x}-\xi)\right|} \\
\left|\sinh \lambda\left(-\frac{1}{2}+\mathrm{i} \frac{\sqrt{3}}{2}\right)(\mathrm{x}-\xi)\right| \leq \mathrm{e}^{\left(-\frac{1}{2} \sigma-\frac{\sqrt{3}}{2} \mathrm{t}\right)(\mathrm{x}-\xi) \mid}
\end{gathered}
$$

where, $\lambda=\sigma+i \tau$ :

$$
\begin{array}{r}
|\cosh \lambda \mathrm{x}| \leq \mathrm{e}^{|\sigma x|},\left|\cosh \lambda\left(\frac{1}{2}+\mathrm{i} \frac{\sqrt{3}}{2}\right) \mathrm{x}\right| \leq \mathrm{e}^{\left(\frac{1}{2} \sigma-\frac{\sqrt{3}}{2} \mathrm{t}\right) \mathrm{x} \mid},\left|\cosh \lambda\left(-\frac{1}{2}+\mathrm{i} \frac{\sqrt{3}}{2}\right) \mathrm{x}\right| \leq \mathrm{e}^{\left(-\frac{1}{2} \sigma-\frac{\sqrt{3}}{2} \mathrm{t}\right) \mathrm{x}} \mid \\
\mathrm{y}_{0}(\mathrm{x}, \lambda) \mid \leq \frac{1}{3}\left[|\cosh \lambda \mathrm{x}|+\left|\cosh \lambda\left(\frac{1}{2}+\mathrm{i} \frac{\sqrt{3}}{2}\right) \mathrm{x}\right|+\cosh \lambda\left(-\frac{1}{2}+\mathrm{i} \frac{\sqrt{3}}{2}\right) \mathrm{x} \mid\right]+\frac{1}{3 \lambda^{5}} \\
\int_{\mathrm{a}}^{\mathrm{x}}\left[|\sinh \lambda(\mathrm{x}-\xi)|+\left(\frac{1}{2}+\mathrm{i} \frac{\sqrt{3}}{2}\right) \sinh \lambda\left(\frac{1}{2}+\mathrm{i} \frac{\sqrt{3}}{2}\right)(\mathrm{x}-\xi)\left|+\left(-\frac{1}{2}+\mathrm{i} \frac{\sqrt{3}}{2}\right) \sinh \lambda\left(-\frac{1}{2}+\mathrm{i} \frac{\sqrt{3}}{2}\right)(\mathrm{x}-\xi)\right|\right] \mathrm{q}(\xi) \mathrm{y}_{0}(\xi) \mathrm{d} \xi
\end{array}
$$

Then:

And since:

$$
\begin{aligned}
& \left|y_{0}(x, \lambda)\right| \leq \frac{1}{3}\left[e^{|\sigma x|}+e^{\left|\left(\frac{1}{2} \sigma-\frac{\sqrt{3}}{2} t\right) x\right|} e_{+e}\left|\left(-\frac{1}{2} \sigma-\frac{\sqrt{3}}{2} t\right) x\right|\right]+\frac{1}{3 \lambda^{5}} \\
& \int_{a}^{x}\left[e^{|\sigma(x-\xi)|}+\left|\left(\frac{1}{2}+i \frac{\sqrt{3}}{2}\right)\right|+e^{\left(\frac{1}{2} \sigma-\frac{\sqrt{3}}{2} t\right)(x-\xi) \mid}|+|\left(-\frac{1}{2}+i \frac{\sqrt{3}}{2}\right) e^{\left(-\frac{1}{2} \sigma-\frac{\sqrt{3}}{2} t\right)(x-\xi)||} \mid\right] \\
& q(\xi) y_{0}(\xi, \lambda) d \xi
\end{aligned}
$$

$$
\begin{aligned}
& \mathrm{e}^{|\sigma x|} \leq \mathrm{e}^{\left(-\frac{1}{2} \sigma-\frac{\sqrt{3}}{2} \mathrm{t}\right) \mathrm{x} \mid}, \mathrm{e}^{\left(\frac{1}{2} \sigma-\frac{\sqrt{3}}{2} \mathrm{t}\right) \mathrm{x} \mid} \leq \mathrm{e}^{\left(-\frac{1}{2} \sigma-\frac{\sqrt{3}}{2} \mathrm{t}\right) \mathrm{x} \mid} \\
& \left.\left|y_{0}(x, \lambda)\right| \leq \frac{1}{3} 3 e^{\mid\left(-\frac{1}{2} \sigma-\frac{\sqrt{3}}{2} t\right)}\right) \mid+\frac{1}{3|\lambda|^{5}} \\
& \int_{a}^{x}\left[\begin{array}{l}
x\left|\left(-\frac{1}{2} \sigma-\frac{\sqrt{3}}{2} t\right)(x-\xi)\right| \\
e^{-\left(-\frac{1}{2} \sigma-\frac{\sqrt{3}}{2} t\right)(x-\xi) \mid}\left|\left(-\frac{1}{2} \sigma-\frac{\sqrt{3}}{2} t\right)(x-\xi)\right|
\end{array}\right]+ \\
& \mathrm{q}(\xi) \mathrm{y}_{0}(\xi, \lambda) \mathrm{d} \xi
\end{aligned}
$$$$
\begin{aligned}
& \left.\left|y_{0}(x, \lambda)\right| \leq e^{\mid\left(-\frac{1}{2} \sigma-\frac{\sqrt{3}}{2} t\right)}\right) \mid+\frac{1}{|\lambda|^{5}} \int_{a}^{x} e^{\left|\left(-\frac{1}{2} \sigma-\frac{\sqrt{3}}{2} t\right)(x-\xi)\right|} \\
& q(\xi) y_{0}(\xi, \lambda) d \xi
\end{aligned}
$$

Put:

$$
\begin{aligned}
& \left|y_{0}(x, \lambda)\right|=\left.e^{\left(-\frac{1}{2} \sigma-\frac{\sqrt{3}}{2} t\right) x}\right|_{f(x, \lambda)} \\
& f(x, \lambda)=y_{0}(x, \lambda) e^{\left|\left(\frac{1}{2} \sigma+\frac{\sqrt{3}}{2} t\right)(x-\xi)\right|}
\end{aligned}
$$




$$
\begin{aligned}
& f(x, \lambda)=y_{0}=\frac{1}{3}\left[\cosh \lambda x+\cosh \lambda\left(\frac{1}{2}+i \frac{\sqrt{3}}{2}\right) x+\cosh \lambda\left(-\frac{1}{2}+i \frac{\sqrt{3}}{2}\right) x\right] e^{\left|\left(\frac{1}{2} \sigma+\frac{\sqrt{3}}{2} t\right)(x-\xi)\right|} \\
& \frac{1}{3 \lambda^{5}} \int_{a}^{x}\left[\sinh \lambda(x-\xi)+\left(\frac{1}{2}+i \frac{\sqrt{3}}{2}\right) \sinh \lambda\left(\frac{1}{2}+i \frac{\sqrt{3}}{2}\right)(x-\xi)+\left(-\frac{1}{2}+i \frac{\sqrt{3}}{2}\right) \sinh \lambda\left(-\frac{1}{2}+i \frac{\sqrt{3}}{2}\right)(x-\xi)\right] \mid e^{\left|\left(\frac{1}{2} \sigma+\frac{\sqrt{3}}{2} t\right)(x-\xi)\right|} \\
& q(\xi) f(\xi, \lambda) d \xi
\end{aligned}
$$

Let, $M(\lambda)$ denote the maximum value of $|f(x, \lambda)|$ for $\mathrm{x}$ in $[\mathrm{a}, \mathrm{b}]$ then we obtain:

$$
\begin{aligned}
& \mathrm{M}(\lambda) \leq 1+\frac{1}{|\lambda|^{5}} \mathrm{M}(\lambda) \int_{\mathrm{a}}^{\mathrm{x}}|\mathrm{q}(\xi)| \mathrm{d} \xi \\
& \mathrm{M}(\lambda)\left(1-\frac{1}{|\lambda|^{5}} \mathrm{M}(\lambda) \int_{\mathrm{a}}^{\mathrm{x}}|\mathrm{q}(\xi)| \mathrm{d} \xi\right) \leq 1 \\
& \mathrm{M}(\lambda) \leq\left\{1-\frac{1}{|\lambda|^{5}} \mathrm{M}(\lambda) \int_{\mathrm{a}}^{\mathrm{x}}|\mathrm{q}(\xi)| \mathrm{d} \xi\right\}^{-1}
\end{aligned}
$$

$|f(x, \lambda)| \leq M(\lambda)=0(1),|\lambda| \rightarrow \infty$ and therefore:

$$
y_{0}(x, \lambda)=0\left\{e^{d\left(-\frac{1}{2} \sigma-\frac{\sqrt{3}}{2} t\right)(x-\xi) \mid}\right\}
$$

So, the integral equation is:

$$
\begin{aligned}
& 0\left\{\left.\frac{1}{\lambda^{5}} \int_{a}^{x} e^{x}\left|\left(-\frac{1}{2} \sigma-\frac{\sqrt{3}}{2} t\right)(x-\xi)\right| e^{\mid\left(-\frac{1}{2} \sigma-\frac{\sqrt{3}}{2} t\right)(\xi-a)}\right|_{q}(\xi) d \xi\right\}= \\
& 0\left\{|\lambda|^{-5} e^{\left(-\frac{1}{2} \sigma-\frac{\sqrt{3}}{2} t\right)(x-a) \mid}\right\}
\end{aligned}
$$

By the same way we get all derivatives.

Corollary 2: For $\mathrm{x}$ in $[\mathrm{a}, \mathrm{b}]$ and $\lambda \neq 0, \mathrm{y}_{1}(\mathrm{x}, \lambda)$ and its derivatives in theorem 1 can be written as:

$$
\begin{aligned}
& \mathrm{y}_{1}=\frac{1}{3 \lambda}\left[\begin{array}{l}
\sinh \lambda(\mathrm{x}-\mathrm{a})+\frac{1}{2}\left(1+\frac{1}{32} \mathrm{i}\right) \sinh \lambda\left(\frac{1}{2}+\mathrm{i} \frac{\sqrt{3}}{2}\right) \\
(\mathrm{x}-\mathrm{a})+\frac{1}{2}\left(-1+\frac{1}{32} \mathrm{i}\right) \sinh \lambda\left(-\frac{1}{2}+\mathrm{i} \frac{\sqrt{3}}{2}\right)(\mathrm{x}-\mathrm{a})
\end{array}\right]+ \\
& 0\left(\frac{1}{\lambda^{6}} \mathrm{e}^{\mathrm{tt}(\mathrm{x}-\mathrm{a})}\right)
\end{aligned}
$$

$$
\begin{aligned}
& \mathrm{y}_{1}^{\prime}=\frac{1}{3} \\
& {\left[\begin{array}{l}
\cosh \lambda(\mathrm{x}-\mathrm{a})+\frac{1}{2}\left(1+\frac{1}{32} \mathrm{i}\right)\left(\frac{1}{2}+\mathrm{i} \frac{\sqrt{3}}{2}\right) \\
\cosh \lambda\left(\frac{1}{2}+\mathrm{i} \frac{\sqrt{3}}{2}\right)(\mathrm{x}-\mathrm{a})+\frac{1}{2} \\
\left(-1+\frac{1}{32} \mathrm{i}\right)\left(-\frac{1}{2}+\mathrm{i} \frac{\sqrt{3}}{2}\right) \cosh \lambda\left(-\frac{1}{2}+\mathrm{i} \frac{\sqrt{3}}{2}\right)(\mathrm{x}-\mathrm{a})
\end{array}\right]+} \\
& 0\left(\frac{1}{\lambda^{5}} \mathrm{e}^{\mid \mathrm{tt}(\mathrm{x}-\mathrm{a})}\right) \\
& \mathrm{y}_{1}^{\prime \prime}=\frac{1}{3} \lambda \\
& {\left[\begin{array}{l}
\sinh \lambda(\mathrm{x}-\mathrm{a})+\frac{1}{2}\left(1+\frac{1}{32} \mathrm{i}\right)\left(\frac{1}{2}+\mathrm{i} \frac{\sqrt{3}}{2}\right)^{2} \sinh \lambda\left(\frac{1}{2}+\mathrm{i} \frac{\sqrt{3}}{2}\right) \\
(\mathrm{x}-\mathrm{a})+\frac{1}{2}\left(-1+\frac{1}{32} \mathrm{i}\right)\left(-\frac{1}{2}+\mathrm{i} \frac{\sqrt{3}}{2}\right)^{2} \sinh \lambda\left(-\frac{1}{2}+\mathrm{i} \frac{\sqrt{3}}{2}\right)(\mathrm{x}-\mathrm{a})
\end{array}\right]+} \\
& 0\left(\frac{1}{\lambda^{4}} \mathrm{e}^{\mid \mathrm{tt}(\mathrm{x}-\mathrm{a})}\right)
\end{aligned}
$$

$$
\begin{aligned}
& \mathrm{y}_{1}^{\prime \prime \prime}=\frac{1}{3} \lambda^{2} \\
& {\left[\begin{array}{l}
\cosh \lambda(\mathrm{x}-\mathrm{a})+\frac{1}{2}\left(1+\frac{1}{32} \mathrm{i}\right)\left(\frac{1}{2}+\mathrm{i} \frac{\sqrt{3}}{2}\right)^{3} \\
\cosh \lambda\left(\frac{1}{2}+\mathrm{i} \frac{\sqrt{3}}{2}\right)(\mathrm{x}-\mathrm{a})+\frac{1}{2}\left(-1+\frac{1}{32} \mathrm{i}\right) \\
\left(-\frac{1}{2}+\mathrm{i} \frac{\sqrt{3}}{2}\right)^{3} \cosh \lambda\left(-\frac{1}{2}+\mathrm{i} \frac{\sqrt{3}}{2}\right)(\mathrm{x}-\mathrm{a})
\end{array}\right]+} \\
& 0\left(\frac{1}{\lambda^{3}} \mathrm{e}^{|\mathrm{t}|(\mathrm{x}-\mathrm{a})}\right)
\end{aligned}
$$

$$
\begin{aligned}
& \mathrm{y}_{1}^{(4)}=\frac{1}{3} \lambda^{3} \\
& {\left[\begin{array}{l}
\sinh \lambda(x-a)+\frac{1}{2}\left(1+\frac{1}{32} \mathrm{i}\right)\left(\frac{1}{2}+\mathrm{i} \frac{\sqrt{3}}{2}\right)^{4} \sinh \lambda\left(\frac{1}{2}+\mathrm{i} \frac{\sqrt{3}}{2}\right) \\
(\mathrm{x}-\mathrm{a})+\frac{1}{2}\left(-1+\frac{1}{32} \mathrm{i}\right)\left(-\frac{1}{2}+\mathrm{i} \frac{\sqrt{3}}{2}\right)^{4} \sinh \lambda\left(-\frac{1}{2}+\mathrm{i} \frac{\sqrt{3}}{2}\right)(\mathrm{x}-\mathrm{a})
\end{array}\right]+} \\
& 0\left(\frac{1}{\lambda^{2}} \mathrm{e}^{\mathrm{tt}(\mathrm{x}-\mathrm{a})}\right)
\end{aligned}
$$




$$
\begin{aligned}
& \mathrm{y}_{1}^{(5)}=\frac{1}{3} \lambda^{4} \\
& {\left[\cosh \lambda(\mathrm{x}-\mathrm{a})+\frac{1}{2}\left(1+\frac{1}{32} \mathrm{i}\right)\left(\frac{1}{2}+\mathrm{i} \frac{\sqrt{3}}{2}\right)^{5} \cosh \lambda\left(\frac{1}{2}+\mathrm{i} \frac{\sqrt{3}}{2}\right)(\mathrm{x}-\mathrm{a})+\frac{1}{2}\left(-1+\frac{1}{32} \mathrm{i}\right)\left(-\frac{1}{2}+\mathrm{i} \frac{\sqrt{3}}{2}\right)^{5} \cosh \lambda\left(-\frac{1}{2}+\mathrm{i} \frac{\sqrt{3}}{2}\right)(\mathrm{x}-\mathrm{a})\right]+} \\
& 0\left(\frac{1}{\lambda} \mathrm{e}^{\mid \mathrm{t}(\mathrm{x}-\mathrm{a})}\right)
\end{aligned}
$$

Corollary 3: For $\mathrm{x}$ in $[\mathrm{a}, \mathrm{b}]$ and $\lambda \neq 0, \mathrm{y}_{2}(\mathrm{x}, \lambda)$ and its derivatives in theorem 1 can be written as:

$$
\begin{aligned}
& y_{2}=\frac{1}{3 \lambda^{2}}\left[\cosh \lambda(x-a)+\frac{1}{2} \cosh \lambda\left(\frac{1}{2}+i \frac{\sqrt{3}}{2}\right)(x-a)+\frac{1}{2} \cosh \lambda\left(-\frac{1}{2}+i \frac{\sqrt{3}}{2}\right)(x-a)\right]+0\left(\frac{1}{\lambda^{7}} e^{|t|(x-a)}\right) \\
& y_{2}^{\prime}=\frac{1}{3 \lambda}\left[\sinh \lambda(x-a)+\frac{1}{2}\left(\frac{1}{2}+i \frac{\sqrt{3}}{2}\right) \sinh \lambda\left(\frac{1}{2}+i \frac{\sqrt{3}}{2}\right)(x-a)+\frac{1}{2}\left(-\frac{1}{2}+i \frac{\sqrt{3}}{2}\right) \sinh \lambda\left(-\frac{1}{2}+i \frac{\sqrt{3}}{2}\right)(x-a)\right]+0\left(\frac{1}{\lambda^{6}} e^{|t|(x-a)}\right) \\
& y_{2}^{\prime \prime}=\frac{1}{3}\left[\cosh \lambda(x-a)+\frac{1}{2}\left(\frac{1}{2}+i \frac{\sqrt{3}}{2}\right)^{2} \cosh \lambda\left(\frac{1}{2}+i \frac{\sqrt{3}}{2}\right)(x-a)+\frac{1}{2}\left(-\frac{1}{2}+i \frac{\sqrt{3}}{2}\right)^{2} \cosh \lambda\left(-\frac{1}{2}+i \frac{\sqrt{3}}{2}\right)(x-a)\right]+0\left(\frac{1}{\lambda^{5}} e^{|t| \mid(x-a)}\right) \\
& y_{2}^{\prime \prime \prime}=\frac{1}{3} \lambda\left[\sinh \lambda(x-a)+\frac{1}{2}\left(\frac{1}{2}+i \frac{\sqrt{3}}{2}\right)^{3} \sinh \lambda\left(\frac{1}{2}+i \frac{\sqrt{3}}{2}\right)(x-a)+\frac{1}{2}\left(-\frac{1}{2}+i \frac{\sqrt{3}}{2}\right)^{3} \sinh \lambda\left(-\frac{1}{2}+i \frac{\sqrt{3}}{2}\right)(x-a)\right]+0\left(\frac{1}{\lambda^{4}} e^{|t|(x-a)}\right) \\
& y_{2}^{(4)}=\frac{1}{3} \lambda^{2}\left[\cosh \lambda(x-a)+\frac{1}{2}\left(\frac{1}{2}+i \frac{\sqrt{3}}{2}\right)^{3} \cosh \lambda\left(\frac{1}{2}+i \frac{\sqrt{3}}{2}\right)(x-a)+\frac{1}{2}\left(-\frac{1}{2}+i \frac{\sqrt{3}}{2}\right)^{3} \cosh \lambda\left(-\frac{1}{2}+i \frac{\sqrt{3}}{2}\right)(x-a)\right]+0\left(\frac{1}{\lambda^{3}} e^{|t| t \mid(x-a)}\right) \\
& y_{2}^{(5)}=\frac{1}{3} \lambda^{3}\left[\sinh \lambda(x-a)+\frac{1}{2}\left(\frac{1}{2}+i \frac{\sqrt{3}}{2}\right)^{4} \sinh \lambda\left(\frac{1}{2}+i \frac{\sqrt{3}}{2}\right)(x-a)+\frac{1}{2}\left(-\frac{1}{2}+i \frac{\sqrt{3}}{2}\right)^{4} \sinh \lambda\left(-\frac{1}{2}+i \frac{\sqrt{3}}{2}\right)(x-a)\right]+0\left(\frac{1}{\lambda^{2}} e^{|t|(x-a)}\right)
\end{aligned}
$$

Corollary 4: For $\mathrm{x}$ in $[\mathrm{a}, \mathrm{b}]$ and $\lambda \neq 0, \mathrm{y}_{3}(\mathrm{x}, \lambda)$ and its derivatives in theorem 1 can be written as:

$$
\begin{gathered}
y_{3}=\frac{1}{3 \lambda^{3}}\left[\sinh \lambda(x-a)-\sinh \lambda\left(\frac{1}{2}+i \frac{\sqrt{3}}{2}\right)(x-a)+\sinh \lambda\left(-\frac{1}{2}+i \frac{\sqrt{3}}{2}\right)(x-a)\right]+0\left(\frac{1}{\lambda^{8}} e^{|t|(x-a)}\right) \\
y_{3}^{\prime}=\frac{1}{3 \lambda^{2}}\left[\cosh \lambda(x-a)-\left(\frac{1}{2}+i \frac{\sqrt{3}}{2}\right) \cosh \lambda\left(\frac{1}{2}+i \frac{\sqrt{3}}{2}\right)(x-a)+\left(-\frac{1}{2}+i \frac{\sqrt{3}}{2}\right) \cosh \lambda\left(-\frac{1}{2}+i \frac{\sqrt{3}}{2}\right)(x-a)\right]+0\left(\frac{1}{\lambda^{7}} e^{|t|(x-a)}\right) \\
y_{3}^{\prime \prime}=\frac{1}{3 \lambda}\left[\sinh \lambda(x-a)-\left(\frac{1}{2}+i \frac{\sqrt{3}}{2}\right)^{2} \sinh \lambda\left(\frac{1}{2}+i \frac{\sqrt{3}}{2}\right)(x-a)+\left(-\frac{1}{2}+i \frac{\sqrt{3}}{2}\right)^{2} \sinh \lambda\left(-\frac{1}{2}+i \frac{\sqrt{3}}{2}\right)(x-a)\right]+ \\
0\left(\frac{1}{\lambda^{6}} e^{|t|(x-a)}\right) \\
y_{3}^{\prime \prime \prime}=\frac{1}{3}\left[\cosh \lambda(x-a)-\left(\frac{1}{2}+i \frac{\sqrt{3}}{2}\right)^{3} \cosh \lambda\left(\frac{1}{2}+i \frac{\sqrt{3}}{2}\right)(x-a)+\left(-\frac{1}{2}+i \frac{\sqrt{3}}{2}\right)^{3} \cosh \lambda\left(-\frac{1}{2}+i \frac{\sqrt{3}}{2}\right)(x-a)\right]+ \\
0\left(\frac{1}{\lambda^{5}} e^{|t|(x-a)}\right)
\end{gathered}
$$




$$
\begin{aligned}
& y_{3}^{(4)}=\frac{1}{3 \lambda}\left[\sinh \lambda(x-a)-\left(\frac{1}{2}+i \frac{\sqrt{3}}{2}\right)^{4} \sinh \lambda\left(\frac{1}{2}+i \frac{\sqrt{3}}{2}\right)(x-a)+\left(-\frac{1}{2}+i \frac{\sqrt{3}}{2}\right)^{4} \sinh \lambda\left(-\frac{1}{2}+i \frac{\sqrt{3}}{2}\right)(x-a)\right]+0\left(\frac{1}{\lambda^{4}} e^{|t|(x-a)}\right) \\
& y_{3}^{(5)}=\frac{1}{3} \lambda^{2}\left[\cosh \lambda(x-a)-\left(\frac{1}{2}+i \frac{\sqrt{3}}{2}\right)^{5} \cosh \lambda\left(\frac{1}{2}+i \frac{\sqrt{3}}{2}\right)(x-a)+\left(-\frac{1}{2}+i \frac{\sqrt{3}}{2}\right)^{5} \cosh \lambda\left(-\frac{1}{2}+i \frac{\sqrt{3}}{2}\right)(x-a)\right]+0\left(\frac{1}{\lambda^{3}} e^{|t| t(x-a)}\right)
\end{aligned}
$$

Corollary 5: For $\mathrm{x}$ in $[\mathrm{a}, \mathrm{b}]$ and $\lambda \neq 0, \mathrm{y}_{4}(\mathrm{x}, \lambda)$ and its derivatives in theorem 1 can be written as:

$$
\begin{aligned}
& \mathrm{y}_{4}=\frac{1}{3 \lambda^{4}}\left[\cosh \lambda(\mathrm{x}-\mathrm{a})+\frac{1}{2}\left(1-\frac{1}{32} \mathrm{i}\right) \cosh \lambda\left(\frac{1}{2}+\mathrm{i} \frac{\sqrt{3}}{2}\right)(\mathrm{x}-\mathrm{a})+\frac{1}{2}\left(-1+\frac{1}{32} \mathrm{i}\right) \cosh \lambda\left(-\frac{1}{2}+\mathrm{i} \frac{\sqrt{3}}{2}\right)(\mathrm{x}-\mathrm{a})\right]+0\left(\frac{1}{\lambda^{9}} \mathrm{e}^{\mid \mathrm{tt}(\mathrm{x}-\mathrm{a})}\right) \\
& \mathrm{y}_{4}^{\prime}=\frac{1}{3 \lambda^{3}}\left[\sinh \lambda(\mathrm{x}-\mathrm{a})+\frac{1}{2}\left(1-\frac{1}{32} \mathrm{i}\right)\left(\frac{1}{2}+\mathrm{i} \frac{\sqrt{3}}{2}\right) \sinh \lambda\left(\frac{1}{2}+\mathrm{i} \frac{\sqrt{3}}{2}\right)(\mathrm{x}-\mathrm{a})+\frac{1}{2}\left(-1+\frac{1}{32} \mathrm{i}\right)\left(-\frac{1}{2}+\mathrm{i} \frac{\sqrt{3}}{2}\right) \sinh \lambda\left(-\frac{1}{2}+\mathrm{i} \frac{\sqrt{3}}{2}\right)(\mathrm{x}-\mathrm{a})\right]+0\left(\frac{1}{\lambda^{8}} \mathrm{e}^{\mathrm{tt|}(\mathrm{x}-\mathrm{a})}\right) \\
& \mathrm{y}_{4}^{\prime \prime}=\frac{1}{3 \lambda^{2}}\left[\cosh \lambda(\mathrm{x}-\mathrm{a})+\frac{1}{2}\left(1-\frac{1}{32} \mathrm{i}\right)\left(\frac{1}{2}+\mathrm{i} \frac{\sqrt{3}}{2}\right)^{2} \cosh \lambda\left(\frac{1}{2}+\mathrm{i} \frac{\sqrt{3}}{2}\right)(\mathrm{x}-\mathrm{a})+\frac{1}{2}\left(-1+\frac{1}{32} \mathrm{i}\right)\left(-\frac{1}{2}+\mathrm{i} \frac{\sqrt{3}}{2}\right)^{2} \cosh \lambda\left(-\frac{1}{2}+\mathrm{i} \frac{\sqrt{3}}{2}\right)(\mathrm{x}-\mathrm{a})\right]+ \\
& 0\left(\frac{1}{\lambda^{7}} \mathrm{e}^{\mid \mathrm{tt|}(\mathrm{x}-\mathrm{a})}\right) \\
& \mathrm{y}_{4}^{\prime \prime \prime}=\frac{1}{3 \lambda}\left[\sinh \lambda(\mathrm{x}-\mathrm{a})+\frac{1}{2}\left(1-\frac{1}{32} \mathrm{i}\right)\left(\frac{1}{2}+\mathrm{i} \frac{\sqrt{3}}{2}\right)^{3} \sinh \lambda\left(\frac{1}{2}+\mathrm{i} \frac{\sqrt{3}}{2}\right)(\mathrm{x}-\mathrm{a})+\frac{1}{2}\left(-1+\frac{1}{32} \mathrm{i}\right)\left(-\frac{1}{2}+\mathrm{i} \frac{\sqrt{3}}{2}\right)^{3} \sinh \lambda\left(-\frac{1}{2}+\mathrm{i} \frac{\sqrt{3}}{2}\right)(\mathrm{x}-\mathrm{a})\right]+ \\
& 0\left(\frac{1}{\lambda^{6}} \mathrm{e}^{\mid \mathrm{tt|}(\mathrm{x}-\mathrm{a})}\right) \\
& \mathrm{y}_{4}^{(4)}=\frac{1}{3}\left[\cosh \lambda(\mathrm{x}-\mathrm{a})+\frac{1}{2}\left(1-\frac{1}{32} \mathrm{i}\right)\left(\frac{1}{2}+\mathrm{i} \frac{\sqrt{3}}{2}\right)^{4} \cosh \lambda\left(\frac{1}{2}+\mathrm{i} \frac{\sqrt{3}}{2}\right)(\mathrm{x}-\mathrm{a})+\frac{1}{2}\left(-1+\frac{1}{32} \mathrm{i}\right)\left(-\frac{1}{2}+\mathrm{i} \frac{\sqrt{3}}{2}\right)^{4} \cosh \lambda\left(-\frac{1}{2}+\mathrm{i} \frac{\sqrt{3}}{2}\right)(\mathrm{x}-\mathrm{a})\right]+ \\
& 0\left(\frac{1}{\lambda^{5}} \mathrm{e}^{|\mathrm{t}|(\mathrm{x}-\mathrm{a})}\right) \\
& \mathrm{y}_{4}^{(5)}=\frac{1}{3} \lambda\left(\sinh \lambda(\mathrm{x}-\mathrm{a})+\frac{1}{2}\left(1-\frac{1}{32} \mathrm{i}\right)\left(\frac{1}{2}+\mathrm{i} \frac{\sqrt{3}}{2}\right)^{5} \sinh \lambda\left(\frac{1}{2}+\mathrm{i} \frac{\sqrt{3}}{2}\right)(\mathrm{x}-\mathrm{a})+\frac{1}{2}\left(-1+\frac{1}{32} \mathrm{i}\right)\left(-\frac{1}{2}+\mathrm{i} \frac{\sqrt{3}}{2}\right)^{5} \sinh \lambda\left(-\frac{1}{2}+\mathrm{i} \frac{\sqrt{3}}{2}\right)(\mathrm{x}-\mathrm{a})\right]+ \\
& \left.\mathrm{e}^{|\mathrm{t}|(\mathrm{x}-\mathrm{a})}\right)
\end{aligned}
$$

Corollary 6: For $\mathrm{x}$ in $[\mathrm{a}, \mathrm{b}]$ and $\lambda \neq 0, \mathrm{y}_{5}(\mathrm{x}, \lambda)$ and its derivatives in theorem 1 can be written as:

$$
\begin{aligned}
& \mathrm{y}_{5}=\frac{1}{3 \lambda^{5}}\left[\sinh \lambda(\mathrm{x}-\mathrm{a})-\frac{1}{2}\left(-1+\frac{1}{32} \mathrm{i}\right) \sinh \lambda\left(\frac{1}{2}+\mathrm{i} \frac{\sqrt{3}}{2}\right)(\mathrm{x}-\mathrm{a})-\frac{1}{2}\left(1+\frac{1}{32} \mathrm{i}\right) \sinh \lambda\left(-\frac{1}{2}+\mathrm{i} \frac{\sqrt{3}}{2}\right)(\mathrm{x}-\mathrm{a})\right]+0\left(\frac{1}{\lambda^{10}} \mathrm{e}^{\mathrm{tt|}(\mathrm{x}-\mathrm{a})}\right) \\
& \mathrm{y}_{5}^{\prime}=\frac{1}{3 \lambda^{4}}\left[\cosh \lambda(\mathrm{x}-\mathrm{a})-\frac{1}{2}\left(-1+\frac{1}{32} \mathrm{i}\right)\left(\frac{1}{2}+\mathrm{i} \frac{\sqrt{3}}{2}\right) \cosh \lambda\left(\frac{1}{2}+\mathrm{i} \frac{\sqrt{3}}{2}\right)(\mathrm{x}-\mathrm{a})-\frac{1}{2}\left(1+\frac{1}{32} \mathrm{i}\right)\left(-\frac{1}{2}+\mathrm{i} \frac{\sqrt{3}}{2}\right) \cosh \lambda\left(-\frac{1}{2}+\mathrm{i} \frac{\sqrt{3}}{2}\right)(\mathrm{x}-\mathrm{a})\right]+ \\
& 0\left(\frac{1}{\lambda^{9}} \mathrm{e}^{\mid \mathrm{tt|}(\mathrm{x}-\mathrm{a})}\right)
\end{aligned}
$$




$$
\begin{aligned}
& y_{5}^{\prime \prime}=\frac{1}{3 \lambda^{3}} \\
& {\left[\begin{array}{l}
\sinh \lambda(\mathrm{x}-\mathrm{a})-\frac{1}{2}\left(-1+\frac{1}{32} \mathrm{i}\right)\left(\frac{1}{2}+\mathrm{i} \frac{\sqrt{3}}{2}\right)^{2} \sinh \lambda\left(\frac{1}{2}+\mathrm{i} \frac{\sqrt{3}}{2}\right) \\
(\mathrm{x}-\mathrm{a})-\frac{1}{2}\left(1+\frac{1}{32} \mathrm{i}\right)\left(-\frac{1}{2}+\mathrm{i} \frac{\sqrt{3}}{2}\right)^{2} \sinh \lambda\left(-\frac{1}{2}+\mathrm{i} \frac{\sqrt{3}}{2}\right)(\mathrm{x}-\mathrm{a})
\end{array}\right]+} \\
& 0\left(\frac{1}{\lambda^{8}} \mathrm{e}^{\mathrm{tt}(\mathrm{x}-\mathrm{a})}\right) \\
& y_{5}^{\prime \prime \prime}=\frac{1}{3 \lambda^{2}} \\
& {\left[\begin{array}{l}
\cosh \lambda(x-a)-\frac{1}{2}\left(-1+\frac{1}{32} \mathrm{i}\right)\left(\frac{1}{2}+\mathrm{i} \frac{\sqrt{3}}{2}\right)^{3} \cosh \lambda\left(\frac{1}{2}+\mathrm{i} \frac{\sqrt{3}}{2}\right) \\
(\mathrm{x}-\mathrm{a})-\frac{1}{2}\left(1+\frac{1}{32} \mathrm{i}\right)\left(-\frac{1}{2}+\mathrm{i} \frac{\sqrt{3}}{2}\right)^{3} \cosh \lambda\left(-\frac{1}{2}+\mathrm{i} \frac{\sqrt{3}}{2}\right)(\mathrm{x}-\mathrm{a})
\end{array}\right]+} \\
& 0\left(\frac{1}{\lambda^{7}} \mathrm{e}^{\mathrm{t} t(\mathrm{x}-\mathrm{a})}\right) \\
& y_{5}^{(4)}=\frac{1}{3 \lambda} \\
& {\left[\begin{array}{l}
\sinh \lambda(\mathrm{x}-\mathrm{a})-\frac{1}{2}\left(-1+\frac{1}{32} \mathrm{i}\right)\left(\frac{1}{2}+\mathrm{i} \frac{\sqrt{3}}{2}\right)^{4} \sinh \lambda\left(\frac{1}{2}+\mathrm{i} \frac{\sqrt{3}}{2}\right) \\
(\mathrm{x}-\mathrm{a})-\frac{1}{2}\left(1+\frac{1}{32} \mathrm{i}\right)\left(-\frac{1}{2}+\mathrm{i} \frac{\sqrt{3}}{2}\right)^{4} \sinh \lambda\left(-\frac{1}{2}+\mathrm{i} \frac{\sqrt{3}}{2}\right)(\mathrm{x}-\mathrm{a})
\end{array}\right]+} \\
& 0\left(\frac{1}{\lambda^{6}} \mathrm{e}^{\mathrm{tt}(\mathrm{x}-\mathrm{a})}\right) \\
& y_{5}^{(5)}=\frac{1}{3}
\end{aligned}
$$

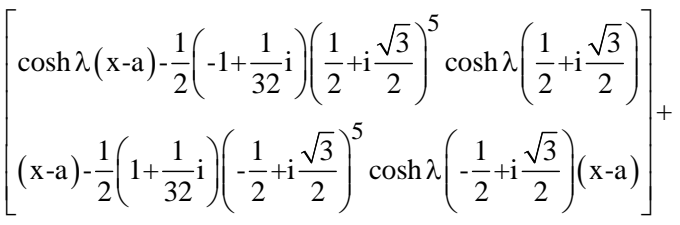

$$
\begin{aligned}
& 0\left(\frac{1}{\lambda^{5}} \mathrm{e}^{\mathrm{lt}(\mathrm{x}-\mathrm{a})}\right)
\end{aligned}
$$

Theorem 2: The formula of eigenfunctions $\psi_{n}(x)$ as $n \rightarrow \infty$ is:

$$
\begin{aligned}
& \psi(\mathrm{x})=\mp \frac{1}{3}\left(\frac{36864}{3071(\mathrm{~b}-\mathrm{a})}\right)^{2} \\
& {\left[\begin{array}{l}
\sinh \lambda(\mathrm{x}-\mathrm{a})+\frac{1}{2}\left(1+\frac{1}{32} \mathrm{i}\right) \sinh \lambda\left(\frac{1}{2}+\mathrm{i} \frac{\sqrt{3}}{2}\right) \\
(\mathrm{x}-\mathrm{a})+\frac{1}{2}\left(-1+\frac{1}{32} \mathrm{i}\right) \sinh \lambda\left(-\frac{1}{2}+\mathrm{i} \frac{\sqrt{3}}{2}\right)(\mathrm{x}-\mathrm{a})
\end{array}\right]+0\left(\frac{1}{\lambda^{2}}\right)}
\end{aligned}
$$

where $\mathrm{a}_{12}=0$ and $\mathrm{a}_{11} \neq 0$.
Proof: If $\psi(\mathrm{x})=\mathrm{c}_{0} \mathrm{y}_{0}+\mathrm{c}_{1} \mathrm{y}_{1}+\mathrm{c}_{2} \mathrm{y}_{2}+\mathrm{c}_{3} \mathrm{y}_{3}+\mathrm{c}_{4} \mathrm{y}_{4}+\mathrm{c}_{5} \mathrm{y}_{5}$ then from first boundary condition we get $\mathrm{a}_{11} \mathrm{c}_{0}+\mathrm{a}_{12} \mathrm{C}_{1}+\mathrm{a}_{13} \mathrm{C}_{2}+\mathrm{a}_{14} \mathrm{C}_{3}+\mathrm{a}_{15} \mathrm{C}_{4}+\mathrm{a}_{16} \mathrm{c}_{5}=0$, since, $\mathrm{c}_{\mathrm{i}}$ cannot be zero for all $i$ then. We can choose $c_{0}=k_{1} a_{12}$ and $\mathrm{c}_{1}=-\mathrm{k}_{1} \mathrm{a}_{11}$ and $\mathrm{c}_{2}=\mathrm{k}_{2} \mathrm{a}_{14}$ and $\mathrm{c}_{3}=-\mathrm{k}_{2} \mathrm{a}_{13}$ and $\mathrm{c}_{4}=\mathrm{k}_{3} \mathrm{a}_{16}$ and $c_{5}=-k_{3} a_{15}$ where $k_{i} \neq 0$ then from theorem 1 we obtain that:

$$
\begin{aligned}
& \psi(x)=\frac{1}{3} k_{1} a_{12}\left[\begin{array}{l}
\cosh \lambda(x-a)+\cosh \lambda\left(\frac{1}{2}+i \frac{\sqrt{3}}{2}\right) \\
(x-a)+\cosh \lambda\left(-\frac{1}{2}+i \frac{\sqrt{3}}{2}\right)(x-a)
\end{array}\right]+0\left(\frac{1}{\lambda^{5}} e^{|t|(x-a)}\right)- \\
& \frac{1}{3 \lambda} \mathrm{k}_{1} \mathrm{a}_{11}\left[\begin{array}{c}
\sinh \lambda(\mathrm{x}-\mathrm{a})+\frac{1}{2}\left(1+\frac{1}{32}\right) \sinh \lambda\left(\frac{1}{2}+\mathrm{i} \frac{\sqrt{3}}{2}\right) \\
(\mathrm{x}-\mathrm{a})+\frac{1}{2}\left(-1+\frac{1}{32}\right) \sinh \lambda\left(-\frac{1}{2}+\mathrm{i} \frac{\sqrt{3}}{2}\right)(\mathrm{x}-\mathrm{a})
\end{array}\right]+ \\
& 0\left(\frac{1}{\lambda^{6}} \mathrm{e}^{|\mathrm{t}|(\mathrm{x}-\mathrm{a})}\right)+\frac{1}{3 \lambda^{2}} \mathrm{k}_{1} \mathrm{a}_{14}\left[\begin{array}{l}
\cosh \lambda(\mathrm{x}-\mathrm{a})+\frac{1}{2} \cosh \lambda\left(\frac{1}{2}+\mathrm{i} \frac{\sqrt{3}}{2}\right) \\
(\mathrm{x}-\mathrm{a})+\frac{1}{2} \cosh \lambda\left(-\frac{1}{2}+\mathrm{i} \frac{\sqrt{3}}{2}\right)(\mathrm{x}-\mathrm{a})
\end{array}\right]+ \\
& 0\left(\frac{1}{\lambda^{7}} \mathrm{e}^{\mid \mathrm{tt}(\mathrm{x}-\mathrm{a})}\right)-\mathrm{k}_{1} \mathrm{a}_{13} \frac{1}{3 \lambda^{3}}\left[\begin{array}{l}
\sinh \lambda(\mathrm{x}-\mathrm{a})-\sinh \lambda\left(\frac{1}{2}+\mathrm{i} \frac{\sqrt{3}}{2}\right) \\
(\mathrm{x}-\mathrm{a})+\sinh \lambda\left(-\frac{1}{2}+\mathrm{i} \frac{\sqrt{3}}{2}\right)(\mathrm{x}-\mathrm{a})
\end{array}\right]+ \\
& 0\left(\frac{1}{\lambda^{8}} \mathrm{e}^{|\mathrm{t}|(\mathrm{x}-\mathrm{a})}\right)-\mathrm{k}_{1} \mathrm{a}_{16} \frac{1}{3 \lambda^{4}}\left[\begin{array}{l}
\cosh \lambda(\mathrm{x}-\mathrm{a})+\frac{1}{2}\left(1-\frac{1}{32} \mathrm{i}\right) \cosh \lambda \\
\left(\frac{1}{2}+\mathrm{i} \frac{\sqrt{3}}{2}\right)(\mathrm{x}-\mathrm{a})+\frac{1}{2}\left(-1+\frac{1}{32} \mathrm{i}\right) \\
\cosh \lambda\left(-\frac{1}{2}+\mathrm{i} \frac{\sqrt{3}}{2}\right)(\mathrm{x}-\mathrm{a})
\end{array}\right]+ \\
& 0\left(\frac{1}{\lambda^{9}} \mathrm{e}^{\mathrm{tt}(\mathrm{x}-\mathrm{a})}\right)-\mathrm{k}_{1} \mathrm{a}_{15} \frac{1}{3 \lambda^{5}}\left[\begin{array}{l}
\sinh \lambda(\mathrm{x}-\mathrm{a})-\frac{1}{2}\left(-1+\frac{1}{32} \mathrm{i}\right) \\
\sinh \lambda\left(\frac{1}{2}+\mathrm{i} \frac{\sqrt{3}}{2}\right)(\mathrm{x}-\mathrm{a})-\frac{1}{2}\left(1+\frac{1}{32} \mathrm{i}\right) \\
\cosh \lambda\left(-\frac{1}{2}+\mathrm{i} \frac{\sqrt{3}}{2}\right)(\mathrm{x}-\mathrm{a})
\end{array}\right]+
\end{aligned}
$$

$0\left(\frac{1}{\lambda^{10}} \mathrm{e}^{\mathrm{t} \mid(\mathrm{x}-\mathrm{a})}\right)$

$$
\begin{aligned}
& \psi(\mathrm{x})=\frac{1}{3} \mathrm{k}_{1} \mathrm{a}_{12} \\
& {\left[\begin{array}{l}
\cosh \lambda(\mathrm{x}-\mathrm{a})+\cosh \lambda\left(\frac{1}{2}+\mathrm{i} \frac{\sqrt{3}}{2}\right) \\
(\mathrm{x}-\mathrm{a})+\cosh \lambda\left(-\frac{1}{2}+\mathrm{i} \frac{\sqrt{3}}{2}\right)(\mathrm{x}-\mathrm{a})
\end{array}\right]+0\left(\frac{1}{\lambda^{5}}\right)}
\end{aligned}
$$




$$
\begin{gathered}
-0\left(\frac{1}{\lambda}\right)+0\left(\frac{1}{\lambda^{6}}\right)+0\left(\frac{1}{\lambda^{2}}\right)+0\left(\frac{1}{\lambda^{7}}\right)-0\left(\frac{1}{\lambda^{3}}\right) \\
+0\left(\frac{1}{\lambda^{8}}\right)+0\left(\frac{1}{\lambda^{4}}\right)+0\left(\frac{1}{\lambda^{9}}\right)-0\left(\frac{1}{\lambda^{5}}\right)+0\left(\frac{1}{\lambda^{10}}\right) \\
\psi(x)=\frac{1}{3} \mathrm{k}_{1} \mathrm{a}_{12}\left[\begin{array}{l}
\left.\cosh \lambda(\mathrm{x}-\mathrm{a})+\cosh \lambda\left(\frac{1}{2}+\mathrm{i} \frac{\sqrt{3}}{2}\right)\right]+0\left(\frac{1}{\lambda}\right) \\
(\mathrm{x}-\mathrm{a})+\cosh \lambda\left(-\frac{1}{2}+\mathrm{i} \frac{\sqrt{3}}{2}\right)(\mathrm{x}-\mathrm{a})
\end{array}\right]
\end{gathered}
$$

If $\mathrm{a}_{12}=0$ then $\mathrm{a}_{11} \neq 0$ :

$$
\psi(x)=-\frac{1}{3 \lambda} \mathrm{k}_{1} \mathrm{a}_{11}\left[\begin{array}{l}
\sinh \lambda(\mathrm{x}-\mathrm{a})+\frac{1}{2}\left(1+\frac{1}{32} \mathrm{i}\right) \\
\sinh \lambda\left(\frac{1}{2}+\mathrm{i} \frac{\sqrt{3}}{2}\right)(\mathrm{x}-\mathrm{a})+\frac{1}{2} \\
\left(-1+\frac{1}{32} \mathrm{i}\right) \sinh \lambda\left(-\frac{1}{2}+\mathrm{i} \frac{\sqrt{3}}{2}\right)(\mathrm{x}-\mathrm{a})
\end{array}\right]+0\left(\frac{1}{\lambda^{2}}\right)
$$

If $\mathrm{a}_{12}=0, \mathrm{a}_{11}=0$ and $\mathrm{a}_{14} \neq 0$ :

$$
\begin{aligned}
& \psi(x)=-\frac{1}{3 \lambda^{4}} k_{3} a_{16} \\
& {\left[\cosh \lambda(x-a)+\frac{1}{2}\left(1-\frac{1}{32} i\right) \cosh \lambda\left(\frac{1}{2}+i \frac{\sqrt{3}}{2}\right)(x-a)+\frac{1}{2}\left(-1+\frac{1}{32} i\right) \cosh \lambda\left(-\frac{1}{2}+i \frac{\sqrt{3}}{2}\right)(x-a)\right]+} \\
& 0\left(\frac{1}{\lambda^{9}} \mathrm{e}^{|t|(x-a)}\right)
\end{aligned}
$$

If $\mathrm{a}_{12}=0, \mathrm{a}_{11}=0, \mathrm{a}_{14}=0, \mathrm{a}_{13}=0, \mathrm{a}_{16}=0$ and $\mathrm{a}_{15} \neq 0$ :

$$
\begin{aligned}
& \psi(\mathrm{x})=-\frac{1}{3 \lambda^{5}} \mathrm{k}_{3} \mathrm{a}_{15} \\
& {\left[\sinh \lambda(\mathrm{x}-\mathrm{a})-\frac{1}{2}\left(-1+\frac{1}{32} \mathrm{i}\right) \sinh \lambda\left(\frac{1}{2}+\mathrm{i} \frac{\sqrt{3}}{2}\right)(\mathrm{x}-\mathrm{a})+\frac{1}{2}\left(1+\frac{1}{32} \mathrm{i}\right) \sinh \lambda\left(-\frac{1}{2}+\mathrm{i} \frac{\sqrt{3}}{2}\right)(\mathrm{x}-\mathrm{a})\right]+} \\
& 0\left(\frac{1}{\lambda^{10}} \mathrm{e}^{\mid \mathrm{t|}(\mathrm{x}-\mathrm{a})}\right)
\end{aligned}
$$

If $\mathrm{a}_{12}=0$ and $\mathrm{a}_{11} \neq 0$ :

$$
\begin{aligned}
& \psi(\mathrm{x})=-\frac{1}{3 \lambda} \mathrm{k}_{1} \mathrm{a}_{11} \\
& {\left[\sinh \lambda(\mathrm{x}-\mathrm{a})+\frac{1}{2}\left(1+\frac{1}{32} \mathrm{i}\right) \sinh \lambda\left(\frac{1}{2}+\mathrm{i} \frac{\sqrt{3}}{2}\right)(\mathrm{x}-\mathrm{a})+\frac{1}{2}\left(-1+\frac{1}{32} \mathrm{i}\right) \sinh \lambda\left(-\frac{1}{2}+\mathrm{i} \frac{\sqrt{3}}{2}\right)(\mathrm{x}-\mathrm{a})\right]-0\left(\frac{1}{\lambda^{2}}\right)}
\end{aligned}
$$

Then :

$$
\begin{aligned}
& \mathrm{k}_{1}^{-1} \psi(\mathrm{x})=-\frac{1}{3 \lambda} \mathrm{a}_{11} \\
& {\left[\sinh \lambda(\mathrm{x}-\mathrm{a})+\frac{1}{2}\left(1+\frac{1}{32} \mathrm{i}\right) \sinh \lambda\left(\frac{1}{2}+\mathrm{i} \frac{\sqrt{3}}{2}\right)(\mathrm{x}-\mathrm{a})+\frac{1}{2}\left(-1+\frac{1}{32} \mathrm{i}\right) \sinh \lambda\left(-\frac{1}{2}+\mathrm{i} \frac{\sqrt{3}}{2}\right)(\mathrm{x}-\mathrm{a})\right]+0\left(\frac{1}{\lambda^{2}}\right)}
\end{aligned}
$$


$\mathrm{k}_{1}^{-2}=\mathrm{k}_{1}^{-2} \int_{\mathrm{a}}^{\mathrm{b}} \psi^{2}(\mathrm{x}) \mathrm{dx}=\frac{1}{9} \mathrm{k}_{1}^{-2} \mathrm{a}_{11}^{2} \int_{\mathrm{a}}^{\mathrm{b}} \frac{1}{\lambda^{2}}\left[\begin{array}{l}\sinh \lambda(\mathrm{x}-\mathrm{a})+\frac{1}{2}\left(1+\frac{1}{32} \mathrm{i}\right) \sinh \lambda\left(\frac{1}{2}+\mathrm{i} \frac{\sqrt{3}}{2}\right) \\ (\mathrm{x}-\mathrm{a})+\frac{1}{2}\left(-1+\frac{1}{32} \mathrm{i}\right) \sinh \lambda\left(-\frac{1}{2}+\mathrm{i} \frac{\sqrt{3}}{2}\right)(\mathrm{x}-\mathrm{a})\end{array}\right]^{2} \mathrm{dx}+0\left(\frac{1}{\lambda^{2}}\right)=$

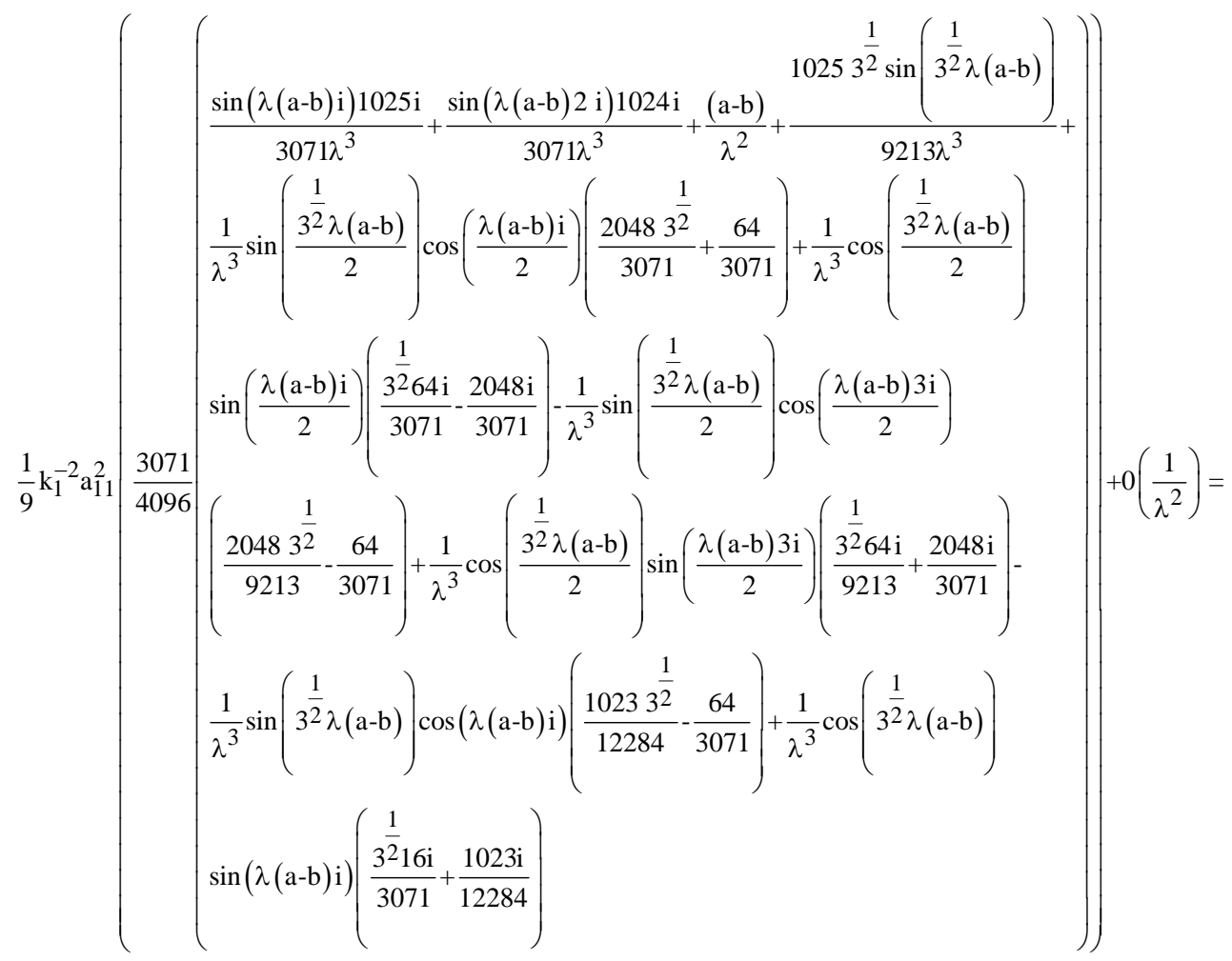
$=\frac{1}{9} \mathrm{k}_{1}^{-2} \mathrm{a}_{11}^{2}\left(\frac{3071}{4096}\left(\begin{array}{l}\left.0\left(\frac{1}{\lambda^{3}}\right)+0\left(\frac{1}{\lambda^{3}}\right)+\frac{(\mathrm{a}-\mathrm{b})}{\lambda^{2}}+0\left(\frac{1}{\lambda^{3}}\right)+0\left(\frac{1}{\lambda^{3}}\right)+0\left(\frac{1}{\lambda^{3}}\right)-\right) \\ 0\left(\frac{1}{\lambda^{3}}\right)+0\left(\frac{1}{\lambda^{3}}\right)-0\left(\frac{1}{\lambda^{3}}\right)+0\left(\frac{1}{\lambda^{3}}\right)+0\left(\frac{1}{\lambda^{3}}\right)\end{array}\right)+0\left(\frac{1}{\lambda^{2}}\right)=\right.$ $\frac{1}{9} \mathrm{k}_{1}^{-2} \mathrm{a}_{11}^{2} \frac{3071}{4096} \frac{(\mathrm{a}-\mathrm{b})}{\lambda^{2}}+0\left(\frac{1}{\lambda^{3}}\right)+0\left(\frac{1}{\lambda^{2}}\right)$

$$
\mathrm{k}_{1}^{-2}=\frac{1}{9} \mathrm{k}_{1}^{-2} \mathrm{a}_{11}^{2} \frac{3071}{4096} \frac{(\mathrm{a}-\mathrm{b})}{\lambda^{2}}+0\left(\frac{1}{\lambda^{2}}\right)
$$

Then:

$$
\begin{aligned}
& a_{11}= \pm \lambda\left(\frac{36864}{3071(b-a)}\right)^{2} \\
& \psi(\mathrm{x})=\mp \frac{1}{3 \lambda} \mathrm{k}_{1} \lambda\left(\frac{36864}{3071(\mathrm{~b}-\mathrm{a})}\right)^{2} \\
& {\left[\begin{array}{l}
\sinh \lambda(\mathrm{x}-\mathrm{a})+\frac{1}{2}\left(1+\frac{1}{32} \mathrm{i}\right) \sinh \lambda\left(\frac{1}{2}+\mathrm{i} \frac{\sqrt{3}}{2}\right) \\
(\mathrm{x}-\mathrm{a})+\frac{1}{2}\left(-1+\frac{1}{32} \mathrm{i}\right) \sinh \lambda\left(-\frac{1}{2}+\mathrm{i} \frac{\sqrt{3}}{2}\right)(\mathrm{x}-\mathrm{a})
\end{array}\right]+} \\
& 0\left(\frac{1}{\lambda^{2}}\right)
\end{aligned}
$$

$$
\begin{aligned}
& \psi(\mathrm{x})=\mp \frac{1}{3}\left(\frac{36864}{3071(\mathrm{~b}-\mathrm{a})}\right)^{2} \\
& {\left[\begin{array}{l}
\sinh \lambda(\mathrm{x}-\mathrm{a})+\frac{1}{2}\left(1+\frac{1}{32} \mathrm{i}\right) \sinh \lambda\left(\frac{1}{2}+\mathrm{i} \frac{\sqrt{3}}{2}\right) \\
(\mathrm{x}-\mathrm{a})+\frac{1}{2}\left(-1+\frac{1}{32} \mathrm{i}\right) \sinh \lambda\left(-\frac{1}{2}+\mathrm{i} \frac{\sqrt{3}}{2}\right)(\mathrm{x}-\mathrm{a})
\end{array}\right]+0\left(\frac{1}{\lambda^{2}}\right)} \\
& \psi(\mathrm{x})=-\frac{1}{3 \lambda^{5}} \mathrm{k}_{3} \mathrm{a}_{15} \\
& {\left[\begin{array}{l}
\sinh \lambda(\mathrm{x}-\mathrm{a})-\frac{1}{2}\left(-1+\frac{1}{32} \mathrm{i}\right) \sinh \lambda\left(\frac{1}{2}+\mathrm{i} \frac{\sqrt{3}}{2}\right) \\
(\mathrm{x}-\mathrm{a})-\frac{1}{2}\left(1+\frac{1}{32} \mathrm{i}\right) \sinh \lambda\left(-\frac{1}{2}+\mathrm{i} \frac{\sqrt{3}}{2}\right)(\mathrm{x}-\mathrm{a})
\end{array}\right]+0\left(\frac{1}{\lambda^{10}}\right)}
\end{aligned}
$$

Then: 


$$
\begin{aligned}
& \mathrm{k}_{3}^{-1} \psi(\mathrm{x})=-\frac{1}{3 \lambda^{5}} \mathrm{a}_{15}\left[\cosh \lambda(\mathrm{x}-\mathrm{a})+\cosh \lambda\left(\frac{1}{2}+\mathrm{i} \frac{\sqrt{3}}{2}\right)(\mathrm{x}-\mathrm{a})+\cosh \lambda\left(-\frac{1}{2}+\mathrm{i} \frac{\sqrt{3}}{2}\right)(\mathrm{x}-\mathrm{a})\right]+0\left(\frac{1}{\lambda^{10}}\right) \\
& \mathrm{k}_{1}^{-2}=\mathrm{k}_{1}^{-2} \int_{\mathrm{a}}^{\mathrm{b}} \psi^{2}(\mathrm{x}) \mathrm{dx}=\frac{1}{9} \mathrm{a}_{15}^{2} \int_{\mathrm{a}}^{\mathrm{b}} \frac{1}{\lambda^{10}}\left[\cosh \lambda(\mathrm{x}-\mathrm{a})+\cosh \lambda\left(\frac{1}{2}+\mathrm{i} \frac{\sqrt{3}}{2}\right)(\mathrm{x}-\mathrm{a})+\cosh \lambda\left(-\frac{1}{2}+\mathrm{i} \frac{\sqrt{3}}{2}\right)(\mathrm{x}-\mathrm{a})\right]^{2} \mathrm{dx}+0\left(\frac{1}{\lambda^{10}}\right)=-\frac{1}{9} \mathrm{a}_{15}^{2} \frac{1}{\lambda^{11}} \\
& \left(-\frac{\sin (\lambda(a-b) i) 1025 i}{4096}-\frac{\sin (\lambda(a-b) 2 i) i}{4}-\frac{\cos \left(3^{\frac{1}{2}} \lambda(a-b)\right) \sin (\lambda(a-b) i) 1023 i \sin \left(3^{\frac{1}{2}} \lambda(a-b)\right) \cos (\lambda(a-b) i)}{16384}+\frac{256}{+}+\right.
\end{aligned}
$$

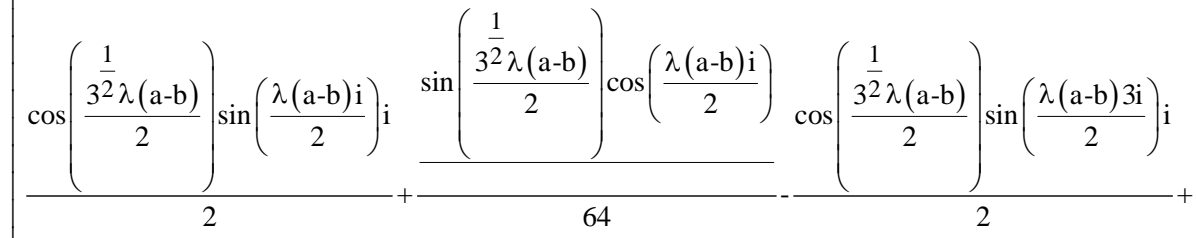

$$
\begin{aligned}
& \frac{\sin \left(\frac{3^{\frac{1}{2}} \lambda(\mathrm{a}-\mathrm{b})}{2}\right) \cos \left(\frac{\lambda(\mathrm{a}-\mathrm{b}) 3 \mathrm{i}}{2}\right) \mathrm{i}}{2} \lambda\left(\frac{3071 \mathrm{a}}{4096}-\frac{3071 \mathrm{~b}}{4096}\right)-\frac{10253^{\frac{1}{2}} \sin \left(3^{\frac{1}{2}} \lambda(\mathrm{a}-\mathrm{b})\right)}{12288}+\frac{3^{\frac{1}{2}} \cos \left(3^{\frac{1}{2}} \lambda(\mathrm{a}-\mathrm{b})\right) \sin (\lambda(\mathrm{a}-\mathrm{b}) \mathrm{i}) \mathrm{i}}{256}++0\left(\frac{1}{\lambda^{10}}\right)
\end{aligned}
$$

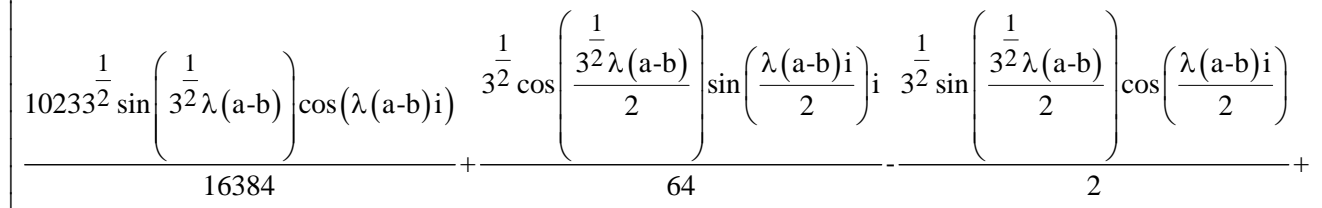

$$
\begin{aligned}
& \frac{3^{\frac{1}{2}} \cos \left(\frac{3^{\frac{1}{2}} \lambda(\mathrm{a}-\mathrm{b})}{2}\right) \sin \left(\frac{\lambda(\mathrm{a}-\mathrm{b}) 3 \mathrm{i}}{2}\right) \mathrm{i} 3^{\frac{1}{2}} \sin \left(\frac{3^{\frac{1}{2}} \lambda(\mathrm{a}-\mathrm{b})}{2}\right) \cos \left(\frac{\lambda(\mathrm{a}-\mathrm{b}) 3 \mathrm{i}}{2}\right)}{62}+\frac{{ }^{2}}{62}
\end{aligned}
$$

Then:

$$
\begin{gathered}
\mathrm{k}_{1}^{-2}=\frac{1}{9} \frac{3071}{4096} \mathrm{a}_{15}^{2} \frac{1}{\lambda^{10}}(\mathrm{a}-\mathrm{b})+0\left(\frac{1}{\lambda^{10}}\right) \\
\text { If } \mathrm{k}_{3}=1 \text { then } \mathrm{a}_{15}= \pm \lambda^{5}\left(\frac{36864}{3071(\mathrm{~b}-\mathrm{a})}\right)^{2} \\
\psi(\mathrm{x})=\mp \frac{1}{3}\left(\frac{36864}{3071(\mathrm{~b}-\mathrm{a})}\right)^{2} \\
{\left[\begin{array}{l}
\sinh \lambda(\mathrm{x}-\mathrm{a})-\frac{1}{2}\left(-1+\frac{1}{32} \mathrm{i}\right) \sinh \lambda\left(\frac{1}{2}+\mathrm{i} \frac{\sqrt{3}}{2}\right) \\
\left.(\mathrm{x}-\mathrm{a})-\frac{1}{2}\left(1+\frac{1}{32} \mathrm{i}\right) \sinh \lambda\left(-\frac{1}{2}+\mathrm{i} \frac{\sqrt{3}}{2}\right)(\mathrm{x}-\mathrm{a})\right]+ \\
0\left(\frac{1}{\lambda^{10}}\right)
\end{array}\right.}
\end{gathered}
$$

Theorem 3: Asymptotic formula for eigen values $\lambda_{\mathrm{n}}$ as $\mathrm{n} \rightarrow \infty$ is:

\section{Proof: Since:}

$$
\lambda=\frac{\mathrm{i} \theta+2 n \pi \mathrm{i}}{\mathrm{b}-\mathrm{a}}
$$

$$
\begin{aligned}
& \Delta(\lambda)= \\
& \left|\begin{array}{llllll}
\mathrm{U}_{0}\left(\mathrm{y}_{0}\right) & \mathrm{U}_{0}\left(\mathrm{y}_{1}\right) & \mathrm{U}_{0}\left(\mathrm{y}_{2}\right) & \mathrm{U}_{0}\left(\mathrm{y}_{3}\right) & \mathrm{U}_{0}\left(\mathrm{y}_{4}\right) & \mathrm{U}_{0}\left(\mathrm{y}_{5}\right) \\
\mathrm{U}_{1}\left(\mathrm{y}_{0}\right) & \mathrm{U}_{1}\left(\mathrm{y}_{1}\right) & \mathrm{U}_{1}\left(\mathrm{y}_{2}\right) & \mathrm{U}_{1}\left(\mathrm{y}_{3}\right) & \mathrm{U}_{1}\left(\mathrm{y}_{4}\right) & \mathrm{U}_{1}\left(\mathrm{y}_{5}\right) \\
\mathrm{U}_{2}\left(\mathrm{y}_{0}\right) & \mathrm{U}_{2}\left(\mathrm{y}_{1}\right) & \mathrm{U}_{2}\left(\mathrm{y}_{2}\right) & \mathrm{U}_{2}\left(\mathrm{y}_{3}\right) & \mathrm{U}_{2}\left(\mathrm{y}_{4}\right) & \mathrm{U}_{2}\left(\mathrm{y}_{5}\right) \\
\mathrm{U}_{3}\left(\mathrm{y}_{0}\right) & \mathrm{U}_{3}\left(\mathrm{y}_{1}\right) & \mathrm{U}_{3}\left(\mathrm{y}_{2}\right) & \mathrm{U}_{3}\left(\mathrm{y}_{3}\right) & \mathrm{U}_{3}\left(\mathrm{y}_{4}\right) & \mathrm{U}_{3}\left(\mathrm{y}_{5}\right) \\
\mathrm{U}_{4}\left(\mathrm{y}_{0}\right) & \mathrm{U}_{4}\left(\mathrm{y}_{1}\right) & \mathrm{U}_{4}\left(\mathrm{y}_{2}\right) & \mathrm{U}_{4}\left(\mathrm{y}_{3}\right) & \mathrm{U}_{4}\left(\mathrm{y}_{4}\right) & \mathrm{U}_{4}\left(\mathrm{y}_{5}\right) \\
\mathrm{U}_{5}\left(\mathrm{y}_{0}\right) & \mathrm{U}_{5}\left(\mathrm{y}_{1}\right) & \mathrm{U}_{5}\left(\mathrm{y}_{2}\right) & \mathrm{U}_{5}\left(\mathrm{y}_{3}\right) & \mathrm{U}_{5}\left(\mathrm{y}_{4}\right) & \mathrm{U}_{5}\left(\mathrm{y}_{5}\right)
\end{array}\right|
\end{aligned}
$$

Then, we can see that: 
J. Eng. Applied Sci., 15 (2): 679-693, 2020

$$
\Delta(\lambda)=\left|\begin{array}{cccccc}
\mathrm{a}_{11} & \mathrm{a}_{12} & \mathrm{a}_{13} & \mathrm{a}_{14} & \mathrm{a}_{15} & \mathrm{a}_{16} \\
\mathrm{a}_{21} & \mathrm{a}_{22} & \mathrm{a}_{23} & \mathrm{a}_{24} & \mathrm{a}_{25} & \mathrm{a}_{26} \\
\mathrm{a}_{31} & \mathrm{a}_{32} & \mathrm{a}_{33} & \mathrm{a}_{34} & \mathrm{a}_{35} & \mathrm{a}_{36} \\
\mathrm{U}_{3}\left(\mathrm{y}_{0}\right) & \mathrm{U}_{3}\left(\mathrm{y}_{1}\right) & \mathrm{U}_{3}\left(\mathrm{y}_{2}\right) \mathrm{U}_{3}\left(\mathrm{y}_{3}\right) & \mathrm{U}_{3}\left(\mathrm{y}_{4}\right) & \mathrm{U}_{3}\left(\mathrm{y}_{5}\right) \\
\mathrm{U}_{4}\left(\mathrm{y}_{0}\right) & \mathrm{U}_{4}\left(\mathrm{y}_{1}\right) & \mathrm{U}_{4}\left(\mathrm{y}_{2}\right) \mathrm{U}_{4}\left(\mathrm{y}_{3}\right) & \mathrm{U}_{4}\left(\mathrm{y}_{4}\right) & \mathrm{U}_{4}\left(\mathrm{y}_{5}\right) \\
\mathrm{U}_{5}\left(\mathrm{y}_{0}\right) & \mathrm{U}_{5}\left(\mathrm{y}_{1}\right) & \mathrm{U}_{5}\left(\mathrm{y}_{2}\right) \mathrm{U}_{5}\left(\mathrm{y}_{3}\right) & \mathrm{U}_{5}\left(\mathrm{y}_{4}\right) & \mathrm{U}_{5}\left(\mathrm{y}_{5}\right)
\end{array}\right|
$$

If we suppose that $X=\left|\begin{array}{lll}a_{13} & a_{15} & a_{16} \\ a_{23} & a_{25} & a_{26} \\ a_{33} & a_{35} & a_{36}\end{array}\right| \neq 0$ and since, the boundary conditions are linearly independent then:

$$
\Delta(\lambda)=\mathrm{X}\left|\begin{array}{lll}
\mathrm{U}_{3}\left(\mathrm{y}_{0}\right) & \mathrm{U}_{3}\left(\mathrm{y}_{1}\right) & \mathrm{U}_{3}\left(\mathrm{y}_{3}\right) \\
\mathrm{U}_{4}\left(\mathrm{y}_{0}\right) & \mathrm{U}_{4}\left(\mathrm{y}_{1}\right) & \mathrm{U}_{4}\left(\mathrm{y}_{3}\right) \\
\mathrm{U}_{5}\left(\mathrm{y}_{0}\right) & \mathrm{U}_{5}\left(\mathrm{y}_{1}\right) & \mathrm{U}_{5}\left(\mathrm{y}_{3}\right)
\end{array}\right|
$$

Then we calculate $U_{j}\left(y_{i}\right)$ for $j=3,4,5$ and $i=0, \ldots$, 5 by using corollary 2-7:

$$
\begin{aligned}
& \mathrm{U}_{3}\left(\mathrm{y}_{0}\right)=\frac{1}{3} \lambda^{5}[3 \cosh (\lambda(\mathrm{a}-\mathrm{b}))-3 \sinh (\lambda(\mathrm{a}-\mathrm{b}))]+0\left(\mathrm{e}^{\mathrm{tt}(\mathrm{x}-\mathrm{a})}\right) \\
& \mathrm{U}_{3}\left(\mathrm{y}_{1}\right)=\frac{1}{3} \lambda^{4}[3 \cosh (\lambda(\mathrm{a}-\mathrm{x}))-3 \sinh (\lambda(\mathrm{a}-\mathrm{x}))]+0\left(\frac{1}{\lambda} \mathrm{e}^{\mid \mathrm{t||}(\mathrm{x}-\mathrm{a})}\right) \\
& \mathrm{U}_{3}\left(\mathrm{y}_{2}\right)=\frac{1}{3} \lambda^{3} \\
& {\left[\begin{array}{l}
3 \cosh (\lambda(a-x))-3 \sinh (\lambda(a-x))+\sin \left(\frac{\frac{1}{32} \lambda(a-x)}{2}\right) \\
\cosh \left(\frac{\lambda(a-x)}{2}\right) i+\frac{1}{4} \cosh \left(\frac{\lambda\left(\frac{1}{32 i}-1\right)(a-x)}{2}\right)\left(\frac{1}{32 i}-1\right)+ \\
\frac{1}{4} \cosh \left(\frac{\lambda\left(\frac{1}{32 i}+1\right)(a-x)}{2}\right)\left(\frac{1}{32 i}-1\right)
\end{array}\right]+} \\
& 0\left(\frac{1}{\lambda^{2}} \mathrm{e}^{\mathrm{tt}(\mathrm{x}-\mathrm{a})}\right) \\
& \mathrm{U}_{3}\left(\mathrm{y}_{3}\right)=\frac{1}{3} \lambda^{2}[3 \cosh (\lambda(\mathrm{a}-\mathrm{x}))-3 \sinh (\lambda(\mathrm{a}-\mathrm{x}))]+0\left(\frac{1}{\lambda^{3}} \mathrm{e}^{|\mathrm{t}|(\mathrm{x}-\mathrm{a})}\right) \\
& \mathrm{U}_{3}\left(\mathrm{y}_{4}\right)=\frac{1}{3} \lambda[3 \cosh (\lambda(\mathrm{a}-\mathrm{x}))-3 \sinh (\lambda(\mathrm{a}-\mathrm{x}))]+0\left(\frac{1}{\lambda^{4}} \mathrm{e}^{\mid \mathrm{t|}(\mathrm{x}-\mathrm{a})}\right) \\
& \mathrm{U}_{3}\left(\mathrm{y}_{5}\right)=\frac{1}{3}[3 \cosh (\lambda(\mathrm{a}-\mathrm{x}))-3 \sinh (\lambda(\mathrm{a}-\mathrm{x}))]+0\left(\frac{1}{\lambda^{5}} \mathrm{e}^{\mathrm{tt}(\mathrm{x}-\mathrm{a})}\right)
\end{aligned}
$$

$$
\begin{aligned}
& \mathrm{U}_{4}\left(\mathrm{y}_{0}\right)=-\frac{1}{3} \lambda^{5}[3 \cosh (\lambda(\mathrm{a}-\mathrm{b}))+3 \sinh (\lambda(\mathrm{a}-\mathrm{b}))]+0\left(\mathrm{e}^{\operatorname{lt}(\mathrm{x}-\mathrm{a})}\right) \\
& \mathrm{U}_{4}\left(\mathrm{y}_{1}\right)=-\frac{1}{3} \lambda^{4}[-3 \cosh (\lambda(\mathrm{a}-\mathrm{x}))-3 \sinh (\lambda(\mathrm{a}-\mathrm{x}))]+ \\
& 0\left(\frac{1}{\lambda} \mathrm{e}^{|t|(x-a)}\right) \\
& \mathrm{U}_{4}\left(\mathrm{y}_{2}\right)=-\frac{1}{3} \lambda^{3} \\
& {\left[3 \cosh (\lambda(a-x))+3 \sinh (\lambda(a-x))-\sin \left(\frac{\frac{1}{32} \lambda(a-x)}{2}\right)\right.} \\
& \cosh \left(\frac{\lambda(a-x)}{2}\right) i+\frac{1}{4} \cosh \left(\frac{\lambda\left(\frac{1}{32 i}-1\right)(a-x)}{2}\right)\left(\frac{1}{32 i}-1\right)++ \\
& {\left[\frac{1}{4} \cosh \left(\frac{\lambda\left(\frac{1}{32 i}+1\right)(a-x)}{2}\right)\left(\frac{1}{32 i}-1\right)\right.} \\
& 0\left(\frac{1}{\lambda^{2}} e^{|t|(x-a)}\right) \\
& \mathrm{U}_{4}\left(\mathrm{y}_{3}\right)=-\frac{1}{3} \lambda^{2}[-3 \cosh (\lambda(\mathrm{a}-\mathrm{x}))-3 \sinh (\lambda(\mathrm{a}-\mathrm{x}))]+ \\
& 0\left(\frac{1}{\lambda^{3}} \mathrm{e}^{|\mathrm{t}|(\mathrm{x}-\mathrm{a})}\right) \\
& \mathrm{U}_{4}(\mathrm{y} 4)=-\frac{1}{3}[\lambda 3 \cosh (\lambda(\mathrm{a}-\mathrm{x}))+3 \sinh (\lambda(\mathrm{a}-\mathrm{x}))]+0\left(\frac{1}{\lambda^{4}} \mathrm{e}^{|\mathrm{t}|(x-a)}\right) \\
& \mathrm{U}_{4}\left(\mathrm{y}_{5}\right)=-\frac{1}{3} \lambda[-3 \cosh (\lambda(\mathrm{a}-\mathrm{x}))-3 \sinh (\lambda(\mathrm{a}-\mathrm{x}))]+0\left(\frac{1}{\lambda^{5}} \mathrm{e}^{\mid \mathrm{tt}(\mathrm{x}-\mathrm{a})}\right) \\
& \mathrm{U}_{5}\left(\mathrm{y}_{0}\right)=\frac{1}{3} \lambda^{5} \\
& {\left[\begin{array}{l}
3 \cosh \frac{(\lambda(\sqrt{3 i}-1)(a-b))}{2}+3 \cosh \frac{(\lambda(\sqrt{3 i}+1)(a-b))}{2}- \\
3 \sinh \frac{(\lambda(\sqrt{3 i}-1)(a-b))}{2}-3 \sinh \frac{(\lambda(\sqrt{3 i}+1)(a-b))}{2}
\end{array}\right]+} \\
& 0\left(e^{|t|(x-a)}\right)
\end{aligned}
$$




$$
\begin{aligned}
& \mathrm{U}_{5}\left(\mathrm{y}_{1}\right)=\frac{1}{3} \lambda^{4}\left[\begin{array}{l}
\cosh \left(\frac{\lambda\left(\frac{1}{32 \mathrm{i}}-1\right)(\mathrm{a}-\mathrm{x})}{2}\right)\left(-\frac{3}{2}+\frac{3 \mathrm{i}}{64}\right)+\frac{1}{4} \cosh \left(\frac{\lambda\left(\frac{1}{32 \mathrm{i}}+1\right)(\mathrm{a}-\mathrm{x})}{2}\right)\left(\frac{3}{2}+\frac{3 \mathrm{i}}{64}\right)+\sinh \\
\left(\frac{\lambda\left(\frac{1}{32 \mathrm{i}}-1\right)(\mathrm{a}-\mathrm{x})}{2}\right)\left(\frac{3}{2}-\frac{3 \mathrm{i}}{64}\right)+\sinh \left(\frac{\lambda\left(\frac{1}{32 \mathrm{i}}+1\right)(\mathrm{a}-\mathrm{x})}{2}\right)\left(-\frac{3}{2}-\frac{3 \mathrm{i}}{64}\right)+
\end{array}\right]+ \\
& 0\left(\frac{1}{\lambda} e^{|t|(x-a)}\right) \\
& \mathrm{U}_{5}\left(\mathrm{y}_{2}\right)=\frac{1}{3} \lambda^{3} \frac{1}{4} \\
& {\left[\begin{array}{l}
-\cosh \left(\frac{\lambda\left(\frac{1}{32 \mathrm{i}}-1\right)(\mathrm{a}-\mathrm{x})}{2}\right)\left(\frac{1}{32 \mathrm{i}}-5\right)-\cosh \left(\frac{\lambda\left(\frac{1}{32 \mathrm{i}}+1\right)(\mathrm{a}-\mathrm{x})}{2}\right)\left(\frac{1}{32 \mathrm{i}}-5\right)+\sinh \left(\frac{\lambda\left(\frac{1}{32 \mathrm{i}}-1\right)(\mathrm{a}-\mathrm{x})}{2}\right) \\
\left(\frac{1}{32 \mathrm{i}}-5\right)+\sinh \left(\frac{\lambda\left(\frac{1}{32 \mathrm{i}}+1\right)(\mathrm{a}-\mathrm{x})}{2}\right)\left(\frac{1}{32 \mathrm{i}}-5\right)
\end{array}\right]+0\left(\frac{1}{\lambda^{2}} \mathrm{e}^{\mathrm{tt}(\mathrm{x}-\mathrm{a})}\right)} \\
& \mathrm{U}_{5}\left(\mathrm{y}_{3}\right)=\frac{1}{3} \lambda^{2} \\
& {\left[3 \cosh \left(\frac{\lambda\left(\frac{1}{32 i}-1\right)(a-x)}{2}\right)-3 \cosh \left(\frac{\lambda\left(\frac{1}{32 i}+1\right)(a-x)}{2}\right)-3 \sinh \left(\frac{\lambda\left(\frac{1}{32 i}-1\right)(a-x)}{2}\right)+\sinh \left(\frac{\lambda\left(\frac{1}{32 i}+1\right)(a-x)}{2}\right)\right]+} \\
& 0\left(\frac{1}{\lambda^{2}} \mathrm{e}^{\mathrm{tt}(\mathrm{x}-\mathrm{a})}\right) \\
& \mathrm{U}_{5}\left(\mathrm{y}_{4}\right)=\frac{1}{3} \lambda \\
& {\left[\begin{array}{l}
\cosh \left(\frac{\lambda\left(\frac{1}{32 \mathrm{i}}-1\right)(\mathrm{a}-\mathrm{x})}{2}\right)\left(-\frac{3}{2}+\frac{3 \mathrm{i}}{64}\right)+\cosh \left(\frac{\lambda\left(\frac{1}{32 \mathrm{i}}+1\right)(\mathrm{a}-\mathrm{x})}{2}\right)\left(\frac{3}{2}+\frac{3 \mathrm{i}}{64}\right)+\sinh \left(\frac{\lambda\left(\frac{1}{32 \mathrm{i}}-1\right)(\mathrm{a}-\mathrm{x})}{2}\right) \\
\left(\frac{3}{2}-\frac{3 \mathrm{i}}{64}\right)+\sinh \left(\frac{\lambda\left(\frac{1}{32 \mathrm{i}}+1\right)(\mathrm{a}-\mathrm{x})}{2}\right)\left(-\frac{3}{2}+\frac{3 \mathrm{i}}{64}\right)
\end{array}\right]+0\left(\frac{1}{\lambda^{4}} \mathrm{e}^{\mathrm{tt}(\mathrm{x}-\mathrm{a})}\right)} \\
& \mathrm{U}_{5}\left(\mathrm{y}_{5}\right)=\frac{1}{3} \\
& {\left[\begin{array}{l}
\cosh \left(\frac{\lambda\left(\frac{1}{32 i}-1\right)(a-x)}{2}\right)\left(-\frac{3}{2}-\frac{3 i}{64}\right)+\cosh \left(\frac{\lambda\left(\frac{1}{32 i}+1\right)(a-x)}{2}\right)\left(\frac{3}{2}-\frac{3 i}{64}\right)+\sinh \left(\frac{\lambda\left(\frac{1}{32 i}-1\right)(a-x)}{2}\right) \\
\left(\frac{3}{2}+\frac{3 i}{64}\right)+\sinh \left(\frac{\lambda\left(\frac{1}{32 i}+1\right)(a-x)}{2}\right)\left(-\frac{3}{2}+\frac{3 i}{64}\right)
\end{array}\right]+0\left(\frac{1}{\lambda^{5}} e^{|t| t(x-a)}\right)}
\end{aligned}
$$




$$
\begin{aligned}
& \Delta(\lambda)=\mathrm{x} \\
& \left(\frac{\lambda^{11}}{27}\left(\begin{array}{l}
\left.\cosh \frac{(\lambda(\sqrt{3 i}-1)(a-x))}{2}(96-i)+\cosh \frac{(\lambda(\sqrt{3 i}+1)(a-x))}{2}(-96-i)+\sinh \frac{(\lambda(\sqrt{3 i}-1)(a-x))}{2}(-96+i)+\right) \\
\sinh \frac{(\lambda(\sqrt{3 i}+1)(a-x))}{2}(96+i)\left(\begin{array}{l}
\cosh \lambda(a-x) \\
+\sinh \lambda(a-x)
\end{array}\right)(\cosh \lambda(a-x)-\sinh \lambda(a-x)
\end{array}\right)\right) \\
& \frac{1}{32}+0\left(\lambda^{6} e^{|t|(x-a)}\right)+0\left(\lambda e^{|t|(x-a)}\right)+0\left(\frac{1}{\lambda^{2}} e^{|t|(x-a)}\right)
\end{aligned}
$$

$$
\begin{aligned}
& \Delta(\lambda)=\frac{27}{32} x \\
& \left(\lambda^{11}\left(\cosh \frac{(\lambda(\sqrt{3 i}-1)(a-x))}{2}(96-i)+\cosh \frac{(\lambda(\sqrt{3 i}+1)(a-x))}{2}(-96-i)+\sinh \frac{(\lambda(\sqrt{3 i}-1)(a-x))}{2}(-96+i)+\sinh \frac{(\lambda(\sqrt{3 i}+1)(a-x))}{2}(96+i)\right)+\right. \\
& 0\left(\lambda^{6} e^{|t|(x-a)}\right)+0\left(\lambda e^{|t|(x-a)}\right)+0\left(\frac{1}{\lambda^{2}} e^{|t|(x-a)}\right) \\
& \Delta(\lambda)=\frac{27}{32} X \mathrm{e}^{\left(-\frac{\sqrt{3} \lambda(\mathrm{a}-\mathrm{b}) \mathrm{i}}{2}\right)} \mathrm{e}^{\left(-\frac{\lambda(\mathrm{a}-\mathrm{b})}{2}\right)} \lambda^{11} \\
& {\left[\left(\left(e^{(\lambda(a-b))}(96-i)-96-i\right)\right)+0\left(\frac{1}{\lambda^{5}} e^{|t| t(x-a)}\right)+0\left(\frac{1}{\lambda^{10}} e^{|t|(x-a)}\right)+0\left(\frac{1}{\lambda^{12}} e^{|t|(x-a)}\right)\right]} \\
& \Delta(\lambda)=\frac{27}{32} X \mathrm{e}^{\left(-\frac{\sqrt{3} \lambda(\mathrm{a}-\mathrm{b}) \mathrm{i}}{2}\right)} \mathrm{e}^{\left(-\frac{\lambda(\mathrm{a}-\mathrm{b})}{2}\right)} \lambda^{11}\left[\left(\left(\mathrm{e}^{(\lambda(\mathrm{a}-\mathrm{b}))}(96-\mathrm{i})-96-\mathrm{i}\right)\right)+0\left(\frac{1}{\lambda^{5}} \mathrm{e}^{3 \mathrm{tt}(\mathrm{b}-\mathrm{a})}\right)\right]
\end{aligned}
$$

If $\Delta(\lambda)=0$ then:

$$
\left(\mathrm{e}^{(\lambda(a-b))}(96-i)-(96+i)+0\left(\frac{1}{\lambda^{5}} e^{3|t|(b-a)}\right)\right)=0
$$

Then $e^{(\lambda(a-b))}(96-i)-(96+i)=0\left(\frac{1}{\lambda^{5}} e^{3 t \mid t(b-a)}\right)$ for large

value of $|\lambda|$ we get:

$$
\mathrm{e}^{(\lambda(\mathrm{a}-\mathrm{b}))}=\frac{96+\mathrm{i}}{96-\mathrm{i}}
$$

If $\theta=\tan ^{-} 1(96+i / 96-i)$ then:

$$
\begin{gathered}
\lambda=\frac{1}{b-a}\left\{\ln \left|\frac{96+i}{96-i}\right|+i \theta+2 n \pi i\right\}, \text { for } n=0, \pm 1, \pm 2, \ldots \\
\lambda=\frac{1}{b-a}\{\ln 1+i \theta+2 n \pi i\}, \text { for } n=0, \pm 1, \pm 2, \ldots \\
\lambda=\frac{i \theta+2 n \pi i}{b-a}, \text { for } n=0, \pm 1, \pm 2, \ldots
\end{gathered}
$$

\section{CONCLUSION}

In mathematics, spectral theory is an inclusive term for theories that extend a single square matrix's eigenvector and eigenvalue theory to a much broader theory of operator structure in a variety of mathematical spaces.

\section{REFERENCES}

Aigunov, G.A., 1996. On the boundedness of orthonormal eigenfunctions of a class of Sturm-Liouville operators with a weight function of unbounded variation on a finite interval. Russ. Math. Surv., Vol. 51, No. 2.

Jwamer, K.H. and G.A. Aigounv, 2010. About uniform limitation of normalized eigenfunctions of t. regge problem in the case of weight functions, satisfying to lipschitz condition. J. Gen. Math. Notes, 1: 115-129.

Jwamer, K.H. and R.Q. Rasul, 2017. Estimations of the upper bound for the eigen-functions of the fourth order boundary value problem with smooth coefficients. Math. Sci. Lett., 6: 67-74. 
Menken, H., 2010. Accurate asymptotic formulas for eigenvalues and eigenfunctions of a boundary-value problem of fourth order. Boundary Value Prob., Vol. 2010, No. 1, 10.1155/2010/720235

Moller, M. and B. Zinsou, 2012. Spectral asymptotics of self-adjoint fourth order boundary value problems with eigenvalue parameter dependent boundary conditions. Boundary Value Prob., Vol, 2012, No. 1. 10.1186/1687-2770-2012-106
Naimark, M.A., 1967. Linear Differential Operators. Part 1. Frederick Ungar Publishing Company, New York, USA., Pages: 144.

Tamarkin, J., 1928. Some general problems of the theory of ordinary linear differential equations and expansion of an arbitrary function in series of fundamental functions. Math. Z., 27: $1-54$. 